\title{
A first integral to the partially averaged Newtonian potential of the three-body problem
}

\author{
Gabriella Pinzari ${ }^{1}$ if
}

Received: 22 September 2018 / Revised: 6 May 2019 / Accepted: 10 May 2019 / Published online: 25 May 2019

(c) The Author(s) 2019

\begin{abstract}
We consider the partial average, i.e. the Lagrange average with respect to just one of the two mean anomalies, of the Newtonian part of the perturbing function in the three-body problem Hamiltonian. We prove that such a partial average exhibits a non-trivial first integral. We show that this integral is fully responsible for certain cancellations in the averaged Newtonian potential, including a property noticed by Harrington in the 1960s. We also highlight its joint role (together with certain symmetries) in the appearance of the so-called Herman resonance. Finally, we discuss an application and an open problem.
\end{abstract}

Keywords Integrable systems · Renormalizable integrability · Harrington property · Herman resonance

Mathematics Subject Classification 34C20 - 70F10 · 37J10 · 37J15 · 37J40

\section{Motivation}

The purpose of this work is to highlight a property of the "partial average of the Newtonian potential" and discuss some consequences.

By "partial averaged Newtonian potential", we mean the following. Let $\left(y^{(i)}, x^{(i)}\right)=$ $\left(\left(y_{1}^{(i)}, y_{2}^{(i)}, y_{3}^{(i)}\right),\left(x_{1}^{(i)}, x_{2}^{(i)}, x_{3}^{(i)}\right)\right)$, with $i=1,2$, be impulse-position coordinates for a two-particle system (which we also call "planets") and let

I wish to thank the Associate Editor and the anonymous Reviewers, who, with their constructive remarks, considerably helped me to improve the presentation of the results. I am indebted to Giacomo Tommei, for providing me reference Bekov and Omarov (1978), which was the starting point of this study. I heartily thank A. Celletti, C. Efthimiopulos, Marcel Guardia, Tere Seara and Lei Zhao for their interest. I am grateful to the Mathematical Sciences Research Institute in Berkeley for its kind hospitality in Fall 2018, during which the paper was written. This research has been funded by the European Research Council (Grant 677793 Stable and Chaotic Motions in the Planetary Problem).

Gabriella Pinzari

gabriella.pinzari@math.unipd.it

1 Dipartimento di Matematica “T. Levi-Civita”, Via Trieste, 63, 35131 Padua, Italy 
$\mathcal{C}: \quad\left(\Lambda_{2}, \ell_{2}, u, v\right) \in \mathcal{A} \times \mathbb{T} \times V \rightarrow(y, x)=\left(y^{(1)}, y^{(2)}, x^{(1)}, x^{(2)}\right) \in\left(\mathbb{R}^{3}\right)^{4}$,

where $\mathcal{A}$ is a domain ${ }^{1}$ in $\mathbb{R}, V$ is a domain in $\mathbb{R}^{10}, \mathbb{T}:=\mathbb{R} /(2 \pi \mathbb{Z}),(u, v)=$ $\left(\left(u_{1}, u_{2}, u_{3}, u_{4}, u_{5}\right),\left(v_{1}, v_{2}, v_{3}, v_{4}, v_{5}\right)\right)$, be a change in coordinates, which we call, for brevity, partial Kepler map, which "preserves the standard two forms":

$$
d y^{(1)} \wedge d x^{(1)}+d y^{(2)} \wedge d x^{(2)}=d \Lambda_{2} \wedge d \ell_{2}+d u \wedge d v
$$

and "integrates the Keplerian motions of $\left(y^{(2)}, x^{(2)}\right)$ ":

$$
\left(\frac{\left\|y^{(2)}\right\|^{2}}{2 \mathrm{~m}_{2}}-\frac{\mathrm{m}_{2} \mathrm{M}_{2}}{\left\|x^{(2)}\right\|}\right) \circ \mathcal{C}=-\frac{\mathrm{m}_{2}^{3} \mathrm{M}_{2}^{2}}{2 \Lambda_{2}^{2}}=: \mathrm{h}_{\mathrm{Kep}}^{(2)}\left(\Lambda_{2}\right),
$$

where $\mathrm{m}_{2}, \mathrm{M}_{2}$ are suitable "mass parameters". Of course, we have assumed that the image of $\mathcal{C}$ in (1) is a domain of $(y, x)$ where the left-hand side of (2) takes negative values. We also assume, throughout the paper, that $(y, x)$ are chosen so that the instantaneous ellipse $\mathbb{E}_{2}$ generated by the two-body Hamiltonian (2) has non-vanishing ${ }^{2}$ eccentricity, so we denote as $\mathrm{P}^{(2)},\left\|\mathrm{P}^{(2)}\right\|=1$, the direction of its perihelion. The angle $\ell_{2}$ will be referred to as "mean anomaly", for uniformity with the name attributed to an analogue angle in the set of the coordinates named after Delaunay [see, e.g. Féjoz (2013) for a definition]. We look at the Lagrange average

$$
h_{2}\left(\Lambda_{2}, u, v\right):=\frac{1}{2 \pi} \int_{\mathbb{T}} \frac{d \ell_{2}}{\left\|x^{(1)}\left(\Lambda_{2}, \ell_{2}, u, v\right)-x^{(2)}\left(\Lambda_{2}, \ell_{2}, u, v\right)\right\|},
$$

which we will refer to as partially averaged Newtonian potential.

There are many examples, in Celestial Mechanics, of canonical maps of the form above. Well-known ones are the above-mentioned Delaunay map (hereafter, $\mathcal{D}$ ), or the coordinates after the Jacobi-Deprit reduction ${ }^{3}$ of the nodes $(\mathcal{J})$ (Jacobi 1842; Deprit 1983). Another example, called "perihelia reduction" $(\mathcal{P})$, has been introduced by Pinzari (2018b). A comprehensive review can be found in Pinzari (2015). All the maps mentioned here might actually be named double Kepler maps, since, in such cases, they satisfy (1)-(2), with, in turn, the $(u, v)$ 's having the form

$$
(u, v)=\left(\Lambda_{1}, \ell_{1}, \hat{u}, \hat{v}\right), \quad d u \wedge d v=d \Lambda_{1} \wedge d \ell_{1}+d \hat{u} \wedge d \hat{v},
$$

where $\ell_{1} \in \mathbb{T}$ and $\Lambda_{1}$ is such that (2) holds also with $\mathrm{m}_{2}, \mathrm{M}_{2}, y^{(2)}, x^{(2)}$ replaced by $\mathrm{m}_{1}, \mathrm{M}_{1}$, $y^{(1)}, x^{(1)}$. In Sect. 2, we present a "genuine" partial Kepler map, namely a map $\mathcal{C}$ where (2) holds only for one of the bodies.

We have been interested to function (3) because, in planetary $(1+N)$-body theories, one has to deal with analogue maps of the kind

$$
\mathcal{C}_{N}: \quad(\Lambda, \ell, \hat{u}, \hat{v}) \in \mathcal{A}^{N} \times \mathbb{T}^{N} \times W \rightarrow\left(\left(y^{(1)}, \ldots, y^{(N)}\right),\left(x^{(1)}, \ldots, x^{(N)}\right)\right),
$$

with $W$ a domain in $\mathbb{R}^{4 N}$ in terms of which the Hamiltonian of the system is

$$
H_{N}(\Lambda, \ell, \hat{u}, \hat{v})=-\sum_{i=1}^{N} \frac{\mathrm{m}_{i}^{3} \mathrm{M}_{i}^{2}}{2 \Lambda_{i}^{2}}+f_{N}(\Lambda, \ell, \hat{u}, \hat{v}),
$$

\footnotetext{
1 By "domain" we mean an open and connected set in $\mathbb{K}=\mathbb{R}^{m}, \mathbb{C}^{m}$.

2 For simplicity, we refrain to formulate the results in the case that the map $\mathcal{C}$ in (1) is regular when the eccentricity of $\mathbb{E}_{2}$ vanishes, as it happens, for example, in the case of the Poincaré or the RPS map.

3 The coordinates discovered by Deprit (1983) are an extension, to any number of particles, of Jacobi (1842), which hold only for a two-particle system.
} 
where the Hamiltonian is composed of a leading "Keplerian part", given by $-\sum_{i=1}^{N} \frac{\mathrm{m}_{i}^{3} \mathrm{M}_{i}^{2}}{2 \Lambda_{i}^{2}}$, slightly perturbed by a function $f_{N}$. The splitting (4) is possible-and in fact it has been adopted by Arnold (1963), Laskar and Robutel (1995), Chierchia and Pinzari (2011), Palacián et al. (2013), Meyer et al. (2018) and Pinzari (2018b), for example, in the so-called planetary problem where one of the masses ("sun") is much larger than the remaining, equally sized, $N$ ones ("planets"). In that case, averaging over the Keplerian frequency vector

$$
\omega_{\mathrm{Kep}}=\left(\omega_{\mathrm{Kep}, 1}, \ldots, \omega_{\mathrm{Kep}, N}\right), \quad \omega_{\mathrm{Kep}, \mathrm{i}}=\frac{\mathrm{m}_{i}^{3} \mathrm{M}_{i}^{2}}{\Lambda_{i}^{3}}
$$

leads to study the so-called secular problem

$$
\bar{H}_{N}(\Lambda, \hat{u}, \hat{v})=-\sum_{i=1}^{N} \frac{\mathrm{m}_{i}^{3} \mathrm{M}_{i}^{2}}{2 \Lambda_{i}^{2}}+\bar{f}_{N}(\Lambda, \hat{u}, \hat{v}),
$$

where the perturbing term is given by "multi-averaged Newtonian potential" (the study of which goes back to Sundman 1916)

$$
\begin{aligned}
\bar{f}_{N}(\Lambda, \hat{u}, \hat{v}):= & -\frac{1}{(2 \pi)^{N}} \sum_{1 \leq i<j \leq N} m_{i} m_{j} \\
& \int_{\mathbb{T}^{N}} \frac{d \ell_{i} d \ell_{j}}{\left\|x^{(i)}\left(\Lambda_{i}, \Lambda_{j}, \ell_{i}, \ell_{j}, \hat{u}, \hat{v}\right)-x^{(j)}\left(\Lambda_{i}, \Lambda_{j}, \ell_{i}, \ell_{j}, \hat{u}, \hat{v}\right)\right\|} .
\end{aligned}
$$

It is known (Gallavotti 1986) that the dynamics of the full problem is well approximated by the one of the secular one as soon as no resonances between the frequencies (5) appear. In case of resonance, for example, in the case $N=2$, with the two planets being much distant one to the other, it is reasonable to expect that a better approximation is obtained replacing the average (6) with the partial average (3). Concretely, it might be challenging to investigate whether there is an application to any of the following regions of motion that have been proposed by Féjoz (2002, p. 310), for the $N=2$ case:

- the planetary region, where the eccentricity of the outer ellipse and both semi-major axes are in a small compact set, and two masses are small compared to the third mass;

- the lunar region, where the masses are in a compact set, and the outer body is far away from the outer two;

- the anti-planetary region, where the outer body ellipse may have a large mass, provided its ellipse is far away from the outer two;

- the anti-lunar region, when the ellipses of the two outer bodies are close, but the corresponding masses are much different.

We now go back to $h_{2}$ in (3). We firstly observe that

Theorem $1 h_{2}$ is integrable by quadratures.

Indeed, $h_{2}$ has six degrees of freedom and possesses, besides itself, the following five commuting ${ }^{4}$ integrals:

\footnotetext{
4 In Hamiltonian mechanics, $f(p, q), g(p, q)$ are said to be Poisson commuting if their Poisson parentheses $\{f, g\}:=\sum \partial_{p} f \partial_{q} g-\partial_{p} g \partial_{q} f$ vanish. Poisson commutation of $f$ and $g$ is equivalent to say that $g$ remains constant along the Hamiltonian motions of $f$.
} 
$\mathrm{I}_{1}:=$ the semi-major axis action $\Lambda_{2}:=\mathrm{m}_{2} \sqrt{\mathrm{M}_{2} a_{2}}$

$\mathrm{I}_{2}:=$ the Euclidean length $\left\|x^{(1)}\right\|$ of $x^{(1)}$;

$\mathrm{I}_{3}:=$ the Euclidean length of the total angular momentum $\mathrm{C}:=\mathrm{C}^{(1)}+\mathrm{C}^{(2)}$, with $\mathrm{C}^{(i)}:=$ $x^{(i)} \times y^{(i)}$, and " $\times$ " denoting skew product;

$\mathrm{I}_{4}:=$ its third component;

$\mathrm{I}_{5}:=$ the projection of the angular momentum $\mathrm{C}^{(2)}$ along the direction $x^{(1)}$.

Indeed, $I_{1}$ is trivially due to the $\ell_{2}$-averaging; $I_{3}$ and $I_{4}$ descend from the invariance by rotations of $h_{2} ; \mathrm{I}_{2}$ and $\mathrm{I}_{5}$ from invariance by rotations around the $x^{(1)}$ axis. Such integrals are independent if $\mathrm{C}^{(1)}$ and $\mathrm{C}^{(2)}$ are not parallel. Otherwise, the problem reduces to be planar; namely, $h_{2}$ has four degrees of freedom, and three independent commuting integrals are obtained neglecting, in the list above, $\mathrm{I}_{4}$ and $\mathrm{I}_{5}$.

Remark 1 The list of independent first integrals to $h_{2}$ is even longer than the one above. For example, in the spatial case, the three components of $x^{(1)}$ and the three components $\mathrm{C}^{(2)}$ are all first integrals. However, the maximum number of commuting first integrals that can be formed with these quantities is four (and the functions $\mathrm{I}_{2}, \mathrm{I}_{3}, \mathrm{I}_{4}$ and $\mathrm{I}_{5}$ are an example of them).

Remark 2 The integrability of $h_{2}$ does not imply that also the partial average of the threebody problem Hamiltonian is so, because this includes also a kinetic term. This is an even different situation compared to the secular problem mentioned above, whose non-integrability is clearly proven, as a consequence of the so-called splitting of separatrices (Féjoz and Guardia 2016).

We now consider the ellipse generated by the "Kepler Hamiltonian" at left-hand side in (2) and denote as $e_{2}:=\sqrt{1-\frac{\mathrm{G}_{2}^{2}}{\Lambda_{2}^{2}}}$ its eccentricity, where $\mathrm{G}_{2}:=\left\|\mathrm{C}^{(2)}\right\|$. Then, let

$$
\mathrm{E}_{0}:=\mathrm{G}_{2}^{2}-\mathrm{m}_{2}^{2} \mathrm{M}_{2} \mathrm{e}_{2} x^{(1)} \cdot \mathrm{P}^{(2)} .
$$

The following fact is a bit more subtle.

Theorem 2 The function $\mathrm{E}_{0}$ is a first integral of $h_{2}$.

Proof The proof of this theorem uses some results from ${ }^{5}$ Pinzari (2018a) that here we recall. We consider the Hamiltonian

$$
\mathrm{J}=\frac{\left\|y^{(2)}\right\|^{2}}{2 \mathrm{~m}_{2}}-\frac{\mathrm{m}_{2} \mathrm{M}_{2}}{\left\|x^{(2)}\right\|}-\frac{\mathrm{m}_{2} \mathrm{M}_{1}}{\left\|x^{(1)}-x^{(2)}\right\|} .
$$

This is the Hamiltonian of one moving particle $\left(y^{(2)}, x^{(2)}\right)$ having mass $\mathrm{m}_{2}$, subject to the gravitational attraction by two fixed particles: $\mathrm{M}_{2}$, at the origin, and $\mathrm{M}_{1}$, at $x^{(1)}$. The Hamiltonian is integrable by quadratures, for having, as first integrals, the function $\mathrm{I}_{5}$ defined above (which trivializes in the case of the planar problem) and the function

$$
\mathrm{E}=\mathrm{E}_{0}+\mathrm{M}_{1} \mathrm{E}_{1},
$$

where $\mathrm{E}_{0}$ is as in (7), while

$$
\mathrm{E}_{1}=\mathrm{m}_{2}^{2} \frac{x^{(1)} \cdot\left(x^{(1)}-x^{(2)}\right)}{\left\|x^{(1)}-x^{(2)}\right\|}
$$

$5 \mathrm{~m}_{2}, \mathrm{M}_{2}, \mathrm{M}_{1}$, correspond to $\mathrm{m}, \mathcal{M}, \mu \mathcal{M}$ in Pinzari (2018a). 
We write $\mathrm{J}$ and $\mathrm{E}$ in terms of a given partial Kepler map, $\mathcal{C}$. We obtain

$$
\mathrm{J}_{\mathcal{C}}=-\frac{\mathrm{m}_{2}^{3} \mathrm{M}_{2}^{2}}{2 \Lambda_{2}^{2}}-\frac{\mathrm{m}_{2} \mathrm{M}_{1}}{\left\|x_{\mathcal{C}}^{(1)}-x_{\mathcal{C}}^{(2)}\right\|}, \quad \mathrm{E}_{\mathcal{C}}=\mathrm{E}_{0, \mathcal{C}}+\mathrm{M}_{1} \mathrm{E}_{1, \mathcal{C}},
$$

where the sub-fix $\mathcal{C}$ denotes the composition with $\mathcal{C}$. The commutation of $\mathrm{J}_{\mathcal{C}}$ and $\mathrm{E}_{\mathcal{C}}$ implies the following relation, which is obtained picking up the terms at the first order in $\mathbf{M}_{1}$ :

$$
\left\{-\frac{\mathrm{m}_{2}^{3} \mathrm{M}_{2}^{2}}{2 \Lambda_{2}^{2}}, \mathrm{E}_{1, \mathcal{C}}\right\}+\left\{-\frac{\mathrm{m}_{2}}{\left\|x_{\mathcal{C}}^{(1)}-x_{\mathcal{C}}^{(2)}\right\|}, \mathrm{E}_{0, \mathcal{C}}\right\}=0 .
$$

Taking the $\ell_{2}$-average of this identity, the first term vanishes itself:

$$
\frac{1}{2 \pi} \int_{\mathbb{T}}\left\{-\frac{\mathrm{m}_{2}^{3} \mathrm{M}_{2}^{2}}{2 \Lambda_{2}^{2}}, \mathrm{E}_{1, \mathcal{C}}\right\} d \ell_{2}=\frac{1}{2 \pi} \frac{\mathrm{m}_{2}^{3} \mathrm{M}_{2}^{2}}{\Lambda_{2}^{3}} \int_{\mathbb{T}} \partial_{\ell_{2}} \mathrm{E}_{1, \mathcal{C}} d \ell_{2} \equiv 0 .
$$

Hence,

$$
0=\frac{1}{2 \pi} \int_{\mathbb{T}}\left\{-\frac{\mathrm{m}_{2}}{\left\|x_{\mathcal{C}}^{(1)}-x_{\mathcal{C}}^{(2)}\right\|}, \mathrm{E}_{0, \mathcal{C}}\right\} d \ell_{2}=\left\{-\mathrm{m}_{2} h_{2}, \mathrm{E}_{0, \mathcal{C}}\right\}
$$

since $\mathrm{E}_{0, \mathcal{C}}$ is $\ell_{2}$-independent. This is the thesis.

In the next sections, we highlight some properties of the partially averaged Newtonian potential that descend from Theorems 1 and 2. More precisely, the paper is organized as follows. In Sect. 2, we show that, as a consequence of Theorem 2, an infinite number of Fourier coefficients in the expansion of $h_{2}$ with respect to the perihelion of its outer planet cancel. This property is a generalization of a fact noticed by S. Harrington in the 1960s, Harrington (1969). To this purpose, we introduce a set of canonical coordinates in terms of which $h_{2}$ and $\mathrm{E}_{0}$ are reduced to one degree of freedom. In Sect. 3, we show that there is an explicit functional dependence between $h_{2}$ and $\mathrm{E}_{0}$. We call this circumstance "renormalizable integrability". The author argues that it might be helpful in the framework of the study of the three-body problem. For example, it would be nice to understand whether fixed points of $E_{0}$, both of elliptic and hyperbolic character, being at the same time fixed points to $h_{2}$ with the same character, might give rise to quasi-periodic motions in the three-body problem, whether hyperbolic equilibria might lead to a splitting of separatrices, etc. Instead of addressing such issues here (which would lead much further than the purposes of this note; see, however, Pinzari (2018a) for an application in this direction), we discuss the relations between level curves and the fixed points of the two functions. Next, we show that, as a consequence of renormalizable integrability and the well-known

Proposition 1 (Keplerian property)

$$
\frac{1}{2 \pi} \int_{\mathbb{T}} \frac{d \ell_{2}}{\left\|x_{\mathcal{C}}^{(2)}\right\|}=\frac{1}{a_{2}} \forall \mathcal{C} .
$$

A linear combination with integer coefficients in a suitable expansion of $h_{2}$ is identically verified. We name it "generalized Herman resonance" since it recalls the well-known Herman resonance in the doubly averaged Newtonian potential (we refer to Abdullah and Albouy (2001) or Féjoz (2004, Proprieté 80) for information on Herman resonance). After proving, in Sect. 4, an algebraic property of the well-known Legendre polynomials (which, roughly, says that a certain average of a Legendre polynomial is still a Legendre polynomial), we establish, 
in Sect. 5, a link between the aforementioned generalized Herman resonance and Herman resonance. In this conclusive section, we also provide a sort of "eccentricity-inclination" expansion at any order for such function and discuss a problem which is left open.

\section{Generalized Harrington property}

In this section, we assume that the map $\mathcal{C}$ in (1) includes, among the $u$ 's, the impulse $u_{1}:=\mathrm{G}_{2}:=\left\|\mathrm{C}^{(2)}\right\|$. We also give $x^{(1)}, x^{(2)}$ the meaning of "interior", "exterior" planet, respectively, because we write formal expansions with respect to $\left\|x^{(1)}\right\|$.

We prove the following

Theorem 3 Fix a domain for $\mathcal{C}$ where $x_{\mathcal{C}}^{(1)} \times \mathrm{C}_{\mathcal{C}}^{(2)}, \mathrm{C}_{\mathcal{C}}^{(2)} \times \mathrm{P}_{\mathcal{C}}^{(2)}$, and $\mathrm{C}_{\mathcal{C}}^{(2)}$ never vanish. Let

$$
h: \quad\left(\Lambda_{2}, u, v\right) \in \mathcal{A} \times V \rightarrow h\left(\Lambda_{2}, u, v\right)
$$

Poisson commute with $\mathrm{E}_{0}$. Assume that $h$ has the form

$$
h=\sum_{n=0}^{\infty} \sum_{m=0}^{+\infty} h_{n m}\left(\Lambda_{2}, u, v\right) \rho^{n} \cos m \varphi
$$

where $\rho\left(\Lambda_{2}, u, v\right):=\left\|x_{\mathcal{C}}^{(1)}\right\|$ and $\varphi\left(\Lambda_{2}, u, v\right)$ is the angle formed by the two vectors $x_{\mathcal{C}}^{(1)} \times$ $\mathrm{C}_{\mathcal{C}}^{(2)}, \mathrm{C}_{\mathcal{C}}^{(2)} \times \mathrm{P}_{\mathcal{C}}^{(2)}$, with respect to the counterclockwise orientation established by $\mathrm{C}_{\mathcal{C}}^{(2)}$. Assume also that $h_{n m}$ depends on $\left(\Lambda_{2}, u, v\right)$ only via the following quantities

$$
\Lambda_{2}, \quad u_{1}=\mathrm{G}_{2}, \quad \Theta:=\frac{x_{\mathcal{C}}^{(1)} \cdot \mathrm{C}_{\mathcal{C}}^{(2)}}{\left\|x_{\mathcal{C}}^{(1)}\right\|},
$$

with $h_{0 m}$ being independent of $u_{1}=\mathrm{G}_{2}$ for all $m \geq 0$. Then

$$
h_{n m}\left(\Lambda_{2}, u, v\right) \equiv 0 \text { if } m \geq \max \{1, n\}, \forall n \geq 0 .
$$

In the case that $h_{n m}=0$ for $n-m$ odd, for $n \geq 1$, the following stronger identities hold:

$$
h_{n m}\left(\Lambda_{2}, u, v\right) \equiv 0 \text { if } m \geq n-1, \quad \forall n \geq 1 .
$$

To prove Theorem 3, we shall need the following

Lemma 1 Let the functions

$$
h(\Gamma, \gamma)=\sum_{n=0}^{\infty} \sum_{m=0}^{\infty} \varepsilon^{n} h_{n m}(\Gamma) \cos m \gamma \quad g(\Gamma, \gamma)=a(\Gamma)+\varepsilon b(\Gamma) \cos \gamma
$$

verify

$$
\{h, g\}_{\Gamma, \gamma}:=\partial_{\Gamma} h \partial_{\gamma} g-\partial_{\Gamma} g \partial_{\gamma} h \equiv 0
$$

and assume that $\partial_{\Gamma} a \not \equiv 0$ and $h_{0 m}$ is independent of $\Gamma$ for all $m \geq 0$. Then $h_{n m}=0$ for all $m \geq \max \{1, n\}$. 
Proof Due to the assumptions of $h$ and $g$, their Poisson parenthesis at left-hand side of (13) is a Fourier series including only sines $\{\sin k \gamma\}_{k \geq 1}$. Projecting (13) over such basis, we obtain the following relations:

$$
\begin{aligned}
m \partial_{\Gamma} a h_{n m}= & -\frac{1}{2}\left((m-1) h_{n-1, m-1}+(m+1) h_{n-1, m+1}\right) \partial_{\Gamma} b \\
& +\frac{1}{2}\left(\partial_{\Gamma} h_{n-1, m-1}-\partial_{\Gamma} h_{n-1, m+1}+\partial_{\Gamma} h_{n-1,0} \delta_{m, 1}\right) b
\end{aligned}
$$

for all $n=0,1, m=1,2, \ldots$, where $\delta_{i j}$ is the Kronecker symbol, and $h_{-1, k}:=0$ for all $k \in \mathbb{Z}$. We now prove that such relations imply $h_{n m} \equiv 0$ for $m \geq \max \{1, n\}$. We proceed by steps.

(i) We prove $h_{0 m}=0$ for $m=1,2, \ldots$. We use (14) with $n=0$ and $m=1,2, \ldots$ :

$$
\begin{aligned}
m \partial_{\Gamma} a h_{0 m}= & -\frac{1}{2}\left((m-1) h_{-1, m-1}+(m+1) h_{-1, m+1}\right) \\
& +\frac{1}{2}\left(\partial_{\Gamma} h_{-1, m-1}-\partial_{\Gamma} h_{-1, m+1}+\partial_{\Gamma} h_{-1,0} \delta_{m, 1}\right) b \\
\equiv & 0 \quad m=1,2, \ldots
\end{aligned}
$$

since $h_{-1, k}=0$ for all $k \in \mathbb{Z}$, as $\partial_{\Gamma} a \not \equiv 0$.

(ii) We prove $h_{1 m}=0$ for $m \geq 1$.

(ii)-a We prove $h_{11}=0$. We use (14) with $n=m=1$. We obtain

$$
\begin{aligned}
\partial_{\Gamma} a h_{11}= & -\frac{1}{2}\left(2 h_{0,2}\right) \partial_{\Gamma} b \\
& +\frac{1}{2}\left(\partial_{\Gamma} h_{00}-\partial_{\Gamma} h_{02}+\partial_{\Gamma} h_{00} \delta_{11}\right) b \\
\equiv & 0
\end{aligned}
$$

since $h_{02}=0$ by (i) and $\partial_{\Gamma} h_{00}=0$ by assumption.

(ii)-b We prove $h_{1 m}=0$ for $m \geq 2$. We use (14) with $n=1, m \geq 2$ :

$$
\begin{aligned}
m \partial_{\Gamma} a h_{1 m}= & -\frac{1}{2}\left((m-1) h_{0, m-1}+(m+1) h_{0, m+1}\right) \partial_{\Gamma} b \\
& +\frac{1}{2}\left(\partial_{\Gamma} h_{0, m-1}-\partial_{\Gamma} h_{0, m+1}+\partial_{\Gamma} h_{0,0} \delta_{m, 1}\right) b \\
\equiv & 0
\end{aligned}
$$

because the first line vanishes by (i), while the second vanishes because, by assumption, $\partial_{\Gamma} h_{0, p}$ for all $p \geq 1$.

(iii) We prove $h_{n m}=0$ for $n \geq 1$ and $m \geq n$. We proceed by induction on $n$. The case $n=1$ has been done in (ii). We assume that it is true for $n \geq 1$ and prove it for $n+1$. We use (14) replacing $n$ with $n+1$ and taking $m \geq n+1$ :

$$
\begin{aligned}
m \partial_{\Gamma} a h_{n+1, m}= & -\frac{1}{2}\left((m-1) h_{n, m-1}+(m+1) h_{n, m+1}\right) \partial_{\Gamma} b \\
& +\frac{1}{2}\left(\partial_{\Gamma} h_{n, m-1}-\partial_{\Gamma} h_{n, m+1}+\partial_{\Gamma} h_{n, 0} \delta_{m, 1}\right) b \\
\equiv & 0 .
\end{aligned}
$$

Here, we have used that for $m \geq n+1, m+1>m-1 \geq n$, so the first line and the two first terms in the second line vanish. The last term also vanishes because $m \geq n+1 \geq 2$, so the Kronecker symbol is zero. The lemma is completely proved. 
We now proceed to prove Theorem 3. To this end, we introduce a specific system of canonical coordinates which will allow us to apply the lemma above.

The $\mathcal{K}$-map Define the "nodes"

$$
v_{0}:=\mathrm{k} \times \mathrm{C}, \quad v_{1}:=\mathrm{C} \times x^{(1)}, \quad v_{2}:=x^{(1)} \times \mathrm{C}^{(2)}, \quad v_{3}:=\mathrm{C}^{(2)} \times \mathrm{P}^{(2)}
$$

and assume that they do not vanish. Denote, as above, as $\mathrm{P}^{(2)}$, with $\left\|\mathrm{P}^{(2)}\right\|=1$ the direction of its perihelion (well defined because the eccentricity does not vanish), $a_{2}$ its semi-major axis, we define the map

$$
\mathcal{K}: \quad\left(\Lambda_{2}, \mathrm{l}_{2}, \mathrm{Z}, \mathrm{G}, \mathrm{R}_{1}, \mathrm{G}_{2}, \Theta, \mathrm{z}, \mathrm{g}_{2}, \mathrm{~g}, \mathrm{r}_{1}, \vartheta\right) \rightarrow\left(y_{\mathcal{K}}^{(1)}, y_{\mathcal{K}}^{(2)}, x_{\mathcal{K}}^{(1)}, x_{\mathcal{K}}^{(2)}\right),
$$

via the relations

$$
\mathcal{K}^{-1}:\left\{\begin{array} { l } 
{ \mathrm { Z } : = \mathrm { C } \cdot \mathrm { k } } \\
{ \mathrm { G } : = \| \mathrm { C } \| } \\
{ \mathrm { R } _ { 1 } : = \frac { y ^ { ( 1 ) } \cdot x ^ { ( 1 ) } } { \| x ^ { ( 1 ) } \| } } \\
{ \Lambda _ { 2 } = \mathrm { m } _ { 2 } \sqrt { \mathrm { M } _ { 2 } a _ { 2 } } } \\
{ \mathrm { G } _ { 2 } : = \| \mathrm { C } ^ { ( 2 ) } \| } \\
{ \mathrm { g } : = \frac { \mathrm { C } ^ { ( 2 ) } \cdot x ^ { ( 1 ) } } { \| x ^ { ( 1 ) } \| } }
\end{array} \quad \left\{\begin{array}{l}
\mathrm{z}:=\alpha_{\mathrm{k}}\left(\mathrm{i}, v_{0}\right) \\
\mathrm{r}_{1}:=\left\|x^{(1)}\right\| \\
\mathrm{l}_{2}:=\text { mean anomaly of } x^{(2)} \text { on } \mathbb{E} \\
\mathrm{g}_{2}:=\alpha_{\mathrm{C}^{(2)}}\left(v_{2}, v_{3}\right) \\
\vartheta:=\alpha_{x^{(1)}}\left(v_{1}, v_{2}\right)
\end{array}\right.\right.
$$

where $(i, j, k)$ is a prefixed reference frame, and for $u, v \in \mathbb{R}^{3}$ lying in the plane orthogonal to a vector $w$ and $\alpha_{w}(u, v)$ denotes the positively oriented angle $(\bmod 2 \pi)$ between $u$ and $v$ (orientation follows the "right-hand rule"). We remark that the planar case corresponds to taking $\Theta=0$ and $\vartheta=\pi$ (prograde case) of $\vartheta=0$ (retrograde case).

The map $\mathcal{K}$ verifies (1)-(2) with $\ell_{2}=1_{2}$ and $u=\left(\mathrm{G}_{2}, \breve{u}\right), v=\left(\mathrm{g}_{2}, \breve{v}\right)$, where $\breve{u}=$ (Z, G, $\left.\mathrm{R}_{1}, \Theta\right), \breve{v}=\left(\mathrm{z}, \mathrm{g}, \mathrm{r}_{1}, \vartheta\right)$. Therefore, $u$ and $v$ are also as claimed in the assumptions of Theorem 3. The canonical character of the coordinates $\mathcal{K}$ is discussed in Pinzari (2018a) and to such paper we refer also for the formula, in terms of $\mathcal{K}$, of the function $\mathrm{E}_{0}$ in (7), which is

$$
\mathrm{E}_{0}=\mathrm{G}_{2}^{2}+\mathrm{m}_{2}^{2} \mathrm{M}_{2} \mathrm{r}_{1} \sqrt{1-\frac{\mathrm{G}_{2}^{2}}{\Lambda_{2}^{2}}} \sqrt{1-\frac{\Theta^{2}}{\mathrm{G}_{2}^{2}}} \cos \mathrm{g}_{2} .
$$

We continue denoting as $h$ the function in the statement expressed in terms of $\mathcal{K}$. It follows from the definitions (15) that $\rho=\mathrm{r}_{1}$ and $\varphi=\mathrm{g}_{2}$, so, by (9), $h$ is given by

$$
h=\sum_{n=0}^{\infty} \sum_{m=0}^{+\infty} \mathrm{r}_{1}^{n} h_{n m}\left(\Lambda_{2}, \Theta, \mathrm{G}_{2}\right) \cos m \mathrm{~g}_{2} .
$$

Here, we have used that, by assumption, the coefficients $h_{n m}$ in this expansion depend only on $\Lambda_{2}, \mathrm{G}_{2}, \Theta$. Therefore, in terms of $\mathcal{K}$, the assumption that $h$ Poisson commutes with $\mathrm{E}_{0}$ reduces to

$$
\left\{h, \mathrm{E}_{0}\right\}_{\left(\mathrm{G}_{2}, \mathrm{~g}_{2}\right)}=\partial_{\mathrm{G}_{2}} h \partial_{\mathrm{g}_{2}} \mathrm{E}_{0}-\partial_{\mathrm{g}_{2}} h \partial_{\mathrm{G}_{2}} \mathrm{E}_{0} \equiv 0 .
$$


Furthermore, with $a=\mathrm{G}_{2}^{2}$, we have $\partial_{\mathrm{G}_{2}} a \not \equiv 0$ and, finally, $h_{0 m}$ is independent of $\mathrm{G}_{2}$ for all $m \geq 0$, being this one of the assumptions of Theorem 3 . We can thus apply Lemma 1 and we obtain that $h_{n m}\left(\mathrm{r}_{1}, \Lambda_{2}, \Theta\right) \equiv 0$ for $m \geq \max \{1, n\}$. The identities (12) trivially follow, under the additional assumption that $h_{n m}=0$ if $n-m$ is odd.

Application of Theorem 3 to the function $h_{2}$ In this section, we discuss the application of Theorem 3 to the function $h_{2}$ in (3). First of all, $h_{2}$ Poisson commutes with $\mathrm{E}_{0}$, as stated by Theorem 2. As in the proof of Theorem 3, we now write $h_{2}$ in terms of the coordinates $\mathcal{K}$ in (15) and we fix a domain as in the statement of the theorem. This map is useful because $\rho=\mathrm{r}_{2}, \varphi=\mathrm{g}_{2}$ and the functions in (10) are coordinates in such system, so we have only to check that $h_{2}$ affords an expansion of the form:

$$
h_{2}=\sum_{n=0}^{\infty} \sum_{m=0}^{+\infty} \mathrm{r}_{1}^{n} h_{2, n m}\left(\Lambda_{2}, \Theta, \mathrm{G}_{2}\right) \cos m \mathrm{~g}_{2},
$$

with $\partial_{\mathrm{G}_{2}} h_{1,0 m}\left(\Lambda_{2}, \Theta, \mathrm{G}_{2}\right) \equiv 0$. We shall also check that, in this summand, only terms with even $n-m$ appear. We observe that, since $h$ commutes with $\mathrm{I}_{1}, \ldots, \mathrm{I}_{5}$, and, by their definitions, such functions are coordinates in the system $\mathcal{K}$ :

$$
\mathrm{I}_{1}=\Lambda_{2}, \quad \mathrm{I}_{2}=\mathrm{r}_{1}, \quad \mathrm{I}_{3}=\mathrm{G}, \quad \mathrm{I}_{4}=\mathrm{Z}, \quad \mathrm{I}_{5}=\Theta,
$$

we have that $h_{2}$ is independent of their conjugate coordinates, respectively, $\ell_{2}, R_{1}, \mathrm{~g}, \mathrm{z}, \vartheta$. The angles $\mathrm{g}, \mathrm{z}$ are themselves first integrals to $h_{2}$ and so we have that $h_{2}$ is also independent of $\mathrm{G}, \mathrm{Z}$. In summary, $h_{2}$ will be a function of $\mathrm{r}_{1}, \Lambda_{2}, \Theta, \mathrm{G}_{2}, \mathrm{~g}_{2}$ only. Now we check that $h_{2}$ affords an expansion of the form (9), with $h_{n m}$ depending only on the quantities (10). As already observed in the proof of Theorem 3 , in terms of the coordinates $\mathcal{K}$, this reduces to check that $h_{2}$, in terms of $\mathcal{K}$, has an expansion of the form (17). To this end, we start from the expansion of the Newtonian potential in Legendre polynomials (see Sect. 4)

$$
\frac{1}{\left\|x^{(1)}-x^{(2)}\right\|}=\sum_{n=0}^{\infty} \mathcal{P}_{n}(t) \frac{\left\|x^{(1)}\right\|^{n}}{\left\|x^{(2)}\right\|^{n+1}} \quad t:=\frac{x^{(1)} \cdot x^{(2)}}{\left\|x^{(1)}\right\|\left\|x^{(2)}\right\|} .
$$

In terms of $\mathcal{K}$, such quantities are given by

$$
\left\|x^{(1)}\right\|=\mathrm{r}_{1}, \quad\left\|x^{(2)}\right\|=a_{2} \frac{\frac{\mathrm{G}_{2}^{2}}{\Lambda_{2}^{2}}}{1+\sqrt{1-\frac{\mathrm{G}_{2}^{2}}{\Lambda_{2}^{2}}} \cos \mathrm{f}_{2}}, \quad t=-\sqrt{1-\frac{\Theta^{2}}{\mathrm{G}_{2}^{2}}} \cos \left(\mathrm{g}_{2}+\mathrm{f}_{2}\right),
$$

where $a_{2}=\frac{\Lambda_{2}^{2}}{\mathrm{~m}_{2}^{2} \mathrm{M}_{2}} ; \mathrm{f}_{2}=\mathrm{f}_{2}\left(\Lambda_{2}, \mathrm{G}_{2}, \mathrm{l}_{2}\right)$ is the true anomaly. The two former expressions are classical; the one for $t$ has been worked out by Pinzari (2018a). Inserting these expressions into (19) and taking the $1_{2}$-average ${ }^{6}$, we have that

$$
h_{2}\left(\mathrm{r}_{1}, \Lambda_{2}, \Theta, \mathrm{G}_{2}, \mathrm{~g}_{2}\right)=\sum_{n=0}^{\infty} h_{2, n}\left(\Lambda_{2}, \Theta, \mathrm{G}_{2}, \mathrm{~g}_{2}\right) \mathrm{r}_{1}^{n},
$$

\footnotetext{
${ }^{6}$ Recall the well-known transition formula [see, e.g. Palacián et al. (2017)] $d \mathrm{l}_{2}=$ $\frac{\frac{\mathrm{G}_{2}^{3}}{\Lambda_{2}^{3}}}{\left(1+\sqrt{1-\frac{\mathrm{G}_{2}^{2}}{\Lambda_{2}^{2}}} \cos \mathrm{f}_{2}\right)^{2}} d \mathrm{f}_{2}$
} 
with

$$
\begin{aligned}
h_{2, n}\left(\Lambda_{2}, \Theta, \mathrm{G}_{2}, \mathrm{~g}_{2}\right)= & \frac{1}{2 \pi a_{2}^{n+1}} \frac{\Lambda_{2}^{2 n-1}}{\mathrm{G}_{2}^{2 n-1}} \int_{\mathbb{T}}\left(1+\sqrt{1-\frac{\mathrm{G}_{2}^{2}}{\Lambda_{2}^{2}}} \cos \mathrm{f}_{2}\right)^{n-1} \\
& \mathcal{P}_{n}\left(-\sqrt{1-\frac{\Theta^{2}}{\mathrm{G}_{2}^{2}}} \cos \left(\mathrm{g}_{2}+\mathrm{f}_{2}\right)\right) d \mathrm{f}_{2} .
\end{aligned}
$$

This expression shows $h_{2, n}\left(\Lambda_{2}, \Theta, \mathrm{G}_{2}, \mathrm{~g}_{2}\right)$ is even in $\mathrm{g}_{2}$ :

$$
h_{2, n}\left(\Lambda_{2}, \Theta,-\mathrm{g}_{2}\right)=h_{2, n}\left(\Lambda_{2}, \Theta, \mathrm{G}_{2}, \mathrm{~g}_{2}\right) \quad \forall \mathrm{g}_{2} \in \mathbb{T},
$$

so, it affords a Fourier expansion

$$
h_{2, n}\left(\Lambda_{2}, \Theta, \mathrm{G}_{2}, \mathrm{~g}_{2}\right)=\sum_{m=0}^{+\infty} h_{2, n m}\left(\Lambda_{2}, \Theta, \mathrm{G}_{2}\right) \cos m \mathrm{~g}_{2},
$$

and the claimed expansion (17) follows. We finally check that $\partial_{\mathrm{G}_{2}} h_{1,0 m} \equiv 0$ for all $m \geq 0$. But this is a consequence of the fact that, for $\mathrm{r}_{1}=0, h_{2}$ reduces to $\frac{1}{2 \pi} \int_{\mathbb{T}} \frac{d \mathrm{l}_{2}}{\left\|x_{\mathcal{K}}^{(2)}\right\|}$, which is $\Gamma$-independent by Proposition 1 . Then the assertion and hence thesis (11) hold. We now check that, in the case of $h_{2}$, one also has $h_{2, n m}=0$ for $n-m$ odd, so, for $n \geq 1$, the stronger identity in (12) holds. Denoting as $c_{n p} \in \mathbb{Q}$ the coefficients in the expansion

$$
\mathcal{P}_{n}(t)=\sum_{p=0}^{n} c_{n p} t^{p}
$$

where, we recall, only $p$ 's having the same parity as $n$ appear (an explicit formula for the $c_{n p}$ 's is available from the first formula in Eq. 53), so that

$$
\mathcal{P}_{n}\left(-\sqrt{1-\frac{\Theta^{2}}{\mathrm{G}_{2}^{2}}} \cos \left(\mathrm{g}_{2}+\mathrm{f}_{2}\right)\right)=(-1)^{n} \sum_{p=0}^{n} c_{n p}\left(1-\frac{\Theta^{2}}{\mathrm{G}_{2}^{2}}\right)^{p / 2} \cos ^{p}\left(\mathrm{~g}_{2}+\mathrm{f}_{2}\right) .
$$

Using the expansion

$$
\begin{aligned}
\cos ^{p}\left(\mathrm{~g}_{2}+\mathrm{f}_{2}\right) & =\left(\cos \mathrm{g}_{2} \cos \mathrm{f}_{2}-\sin \mathrm{g}_{2} \sin \mathrm{f}_{2}\right)^{p} \\
& =\sum_{k=0}^{p}(-1)^{k}\left(\begin{array}{l}
p \\
k
\end{array}\right) \sin ^{k} \mathrm{~g}_{2} \cos ^{p-k} \mathrm{~g}_{2} \sin ^{k} \mathrm{f}_{2} \cos ^{p-k} \mathrm{f}_{2}
\end{aligned}
$$

and finally inserting this expression into (24) and afterwards into (21), we can write (21) as a trigonometric polynomial in $\mathrm{g}_{2}$ having degree $n$ given by

$$
h_{2, n}=(-1)^{n} \sum_{p=0}^{n} \sum_{k=0}^{p} c_{n p} \hat{h}_{n p k}\left(\Lambda_{2}, \mathrm{G}_{2}, \Theta\right) \sin ^{k} \mathrm{~g}_{2} \cos ^{p-k} \mathrm{~g}_{2},
$$

where

$$
\begin{aligned}
\hat{h}_{n p k}\left(\Lambda_{2}, \mathrm{G}_{2}, \Theta\right)= & (-1)^{k}\left(1-\frac{\Theta^{2}}{\mathrm{G}_{2}^{2}}\right)^{p / 2}\left(\begin{array}{l}
p \\
k
\end{array}\right) \frac{1}{2 \pi a_{2}^{n+1}} \\
& \int_{\mathbb{T}} \sin ^{k} \mathrm{f}_{2} \cos ^{p-k} \mathrm{f}_{2}\left(1+\sqrt{1-\frac{\mathrm{G}_{2}^{2}}{\Lambda_{2}^{2}}} \cos \mathrm{f}_{2}\right)^{n-1} d \mathrm{f}_{2} .
\end{aligned}
$$


The function under the integral in the expression above has the same parity as $k$, so $\hat{h}_{n p k}$ vanishes for $k$ odd. $^{7}$ Therefore, in the summand in (25) only even indices $k$ appear. But for any even $k, \sin ^{k} \mathrm{~g}_{2} \cos ^{p-k} \mathrm{~g}_{2}$ has a Fourier expansion $\sum_{m=0}^{p} b_{m} \cos m \mathrm{~g}_{2}$ where $m$ has the same parity as $p$, which is the same as $n$. We collect all of the information in the following.

Proposition 2 All the assumptions of Theorem 3 are verified with $h=h_{2}$. Therefore, the coefficients $h_{2, n m}$ in the expansion (9) verify (11) and, for $n \geq 1$, they verify the stronger identity (12). Choosing $\mathcal{C}=\mathcal{K}$, the expansion in (20)-(23) holds true, with $h_{2, n m}$ verifying (11) and (12). In particular, the term $h_{2,1}$ vanishes identically and $h_{2,2}$, called the dipolar term, does not depend on $\mathrm{g}_{2}$.

One could ask what the last assertion becomes when using, instead of the $\mathcal{K}$-map, one of the more familiar maps, $\mathcal{D}, \mathcal{J}$ or $\mathcal{P}$, mentioned in the introduction. As a matter of fact, the same assertion holds, apart from parity in the Fourier expansion:

Proposition 3 Let $\mathrm{g}_{2}^{\mathcal{D}}, \mathrm{g}_{2}^{\mathcal{J}}$ or $\mathrm{g}_{2}^{\mathcal{P}}$ denote the angles conjugate to $\mathrm{G}_{2}$, in the case of the maps $\mathcal{D}, \mathcal{J}$ or $\mathcal{P}$. In the expansion

$$
h_{2}=\sum_{n=0}^{+\infty} h_{2, n}\left\|x_{\mathcal{C}}^{(1)}\right\|^{n} \quad \mathcal{C}=\mathcal{D}, \mathcal{J}, \mathcal{P},
$$

the coefficients $h_{2, n}$ afford a Fourier expansion $\sum_{m=0}^{+\infty}\left(a_{n m} \cos \mathrm{g}_{2}^{\mathcal{C}}+b_{n m} \sin \mathrm{g}_{2}^{\mathcal{C}}\right)$, with $m$ having the parity as $n$ and $a_{n m}, b_{n m}$ verifying (11) and (12). In particular, $h_{1,1} \equiv 0$ and $h_{1,2}$ does not depend on $\mathrm{g}_{2}^{\mathcal{D}}, \mathrm{g}_{2}^{\mathcal{J}}$ or $\mathrm{g}_{2}^{\mathcal{P}}$, respectively.

Proof The maps $\mathcal{D}, \mathcal{J}$ or $\mathcal{P}$ share the property that $u_{1}=\mathrm{G}_{2}$ is one of their impulses. However, the coordinate conjugate to $G_{2}$ is different in any of such cases and is given by the angle that here we denote as $\mathrm{g}_{2}^{\mathcal{D}}, \mathrm{g}_{2}^{g}$ or $\mathrm{g}_{2}^{\mathcal{P}}$, formed by a certain "node" (we call so a non-vanishing vector in $\mathbb{R}^{3}$ ) with $\mathrm{P}^{(2)}$ in the plane orthogonal to $\mathrm{C}^{(2)}$, with respect to the positive direction determined by $\mathrm{C}^{(2)}$. The mentioned node is given by:

$$
v_{\mathcal{C}}= \begin{cases}\mathrm{k} \times \mathrm{C}^{(2)} & \text { if } \mathcal{C}=\mathcal{D} \\ \mathrm{C} \times \mathrm{C}^{(2)} & \text { if } \mathcal{C}=\mathcal{J} \\ \mathrm{P}^{(1)} \times \mathrm{C}^{(2)} & \text { if } \mathcal{C}=\mathcal{P}\end{cases}
$$

where $\mathrm{P}^{(1)}$ denotes the direction of the perihelion associated to the Keplerian ellipse of the inner body. We then find the following relation

$$
\mathrm{g}_{2}=\mathrm{g}_{2}^{\mathcal{C}}+\varphi^{\mathcal{C}} \quad \mathcal{C}=\mathcal{D}, \mathcal{J}, \mathcal{P},
$$

where $\varphi^{\mathcal{C}}$ is the angle determined by $\nu_{\mathcal{C}}$ and $\nu_{2}$ in (15). Such function does not depend on $\mathrm{g}_{2}^{\mathcal{C}}$. Since the functions $a_{2}, \Theta, \mathrm{r}_{1}, \zeta_{2}, \mathrm{f}_{2}$ in (20), expressed in terms of $\mathcal{D}, \mathcal{J}, \mathcal{P}$, even do not

7 Incidentally, by explicit computation of the integral, we obtain, for even $k$,

$$
\begin{aligned}
\hat{h}_{n p k}\left(\Lambda_{2}, \mathrm{G}_{2}, \Theta\right)= & (-1)^{k}\left(1-\frac{\Theta^{2}}{\mathrm{G}_{2}^{2}}\right)^{p / 2}\left(\begin{array}{c}
p \\
k
\end{array}\right) \frac{1}{2 \pi a_{2}^{n+1}} \\
& \sum_{j=0}^{n-1} \sum_{r=0}^{k / 2}(-1)^{r}\left(\begin{array}{c}
n-1 \\
j
\end{array}\right)\left(\begin{array}{c}
k / 2 \\
r
\end{array}\right)\left(1-\frac{\mathrm{G}_{2}^{2}}{\Lambda_{2}^{2}}\right)^{j / 2} \frac{(j+p-k+2 r-1) ! !}{(j+p-k+2 r) ! !},
\end{aligned}
$$

where only terms with $j$ having the same parity as $p$ appear. 
depend on $\mathrm{g}_{2}^{\mathcal{C}}$, the proof of Proposition 3 follows, replacing such functions into (20), and using the information given by Proposition 2 .

\section{Renormalizable integrability}

Another consequence of Theorems 1 and 2 is that there actually exists a functional dependence between $h_{2}$ and $\mathrm{E}_{0}$ which we shall write explicitly. To this end, we premise some abstract consideration.

Definition 1 Let $h, g$ be two (commuting) functions of the form

$$
h(p, q, y, x)=\widehat{h}(\mathrm{I}(p, q), y, x), \quad g(p, q, y, x)=\widehat{g}(\mathrm{I}(p, q), y, x),
$$

where

$$
(p, q, y, x) \in \mathcal{D}:=\mathcal{B} \times U,
$$

with $U \subset \mathbb{R}^{2}, \mathcal{B} \subset \mathbb{R}^{2 n}$ open and connected, $(p, q)=\left(p_{1}, \ldots, p_{n}, q_{1}, \ldots, q_{n}\right)$ conjugate coordinates with respect to the two-form $\omega=d y \wedge d x+\sum_{i=1}^{n} d p_{i} \wedge d q_{i}$ and $\mathrm{I}(p, q)=$ $\left(\mathrm{I}_{1}(p, q), \ldots, \mathrm{I}_{n}(p, q)\right)$, with

$$
\mathrm{I}_{i}: \mathcal{B} \rightarrow \mathbb{R}, \quad i=1, \ldots, n
$$

pairwise Poisson commuting:

$$
\left\{\mathrm{I}_{i}, \mathrm{I}_{j}\right\}=0 \quad \forall 1 \leq i<j \leq n \quad i=1, \ldots, n .
$$

We say that $h$ is renormalizably integrable via $g$ if there exists a function

$$
\tilde{h}: \quad \mathrm{I}(\mathcal{B}) \times g(U) \rightarrow \mathbb{R},
$$

such that

$$
h(p, q, y, x)=\widetilde{h}(\mathrm{I}(p, q), \widehat{g}(\mathrm{I}(p, q), y, x))
$$

for all $(p, q, y, x) \in \mathcal{D}$.

Proposition 4 If $h$ is renormalizably integrable via $g$, then:

(i) $I_{1}, \ldots, I_{n}$ are first integrals to $h$ and $g$;

(ii) $h$ and $g$ Poisson commute.

Proof It follows from (26) that

$$
\{h, g\}=\sum_{1 \leq i<j \leq n}\left\{\mathrm{I}_{i}, \mathrm{I}_{j}\right\}\left(\partial_{\mathrm{I}_{i}} \widehat{h} \partial_{\mathrm{I}_{j}} \widehat{g}-\partial_{\mathrm{I}_{i}} \widehat{g} \partial_{\mathrm{I}_{j}} \widehat{h}\right)+\left(\partial_{y} h \partial_{x} g-\partial_{y} g \partial_{x} h\right) .
$$

In this expression, all the terms in the summand vanish because of (28), while the last term vanishes because of (29):

$$
\partial_{y} h \partial_{x} g-\partial_{y} g \partial_{x} h=\partial_{g} \widetilde{h} \partial_{x} g \partial_{y} g-\partial_{y} g \partial_{g} \widetilde{h} \partial_{x} g=0 .
$$

This proves (ii). (i) follows from (ii), replacing the couple $(h, g)$ with $\left(h, \mathrm{I}_{i}\right)$ or $\left(g, \mathrm{I}_{i}\right)$, with $i=1, \ldots, n$.

At level of motion, renormalizable integrability can be rephrased as follows. 
Proposition 5 Let $h$ be renormalizably integrable via g. Fix a value $\mathrm{I}_{0}$ for the integrals $\mathrm{I}$ and look at the motion of $(y, x)$ under $h$ and $g$, on the manifold $\mathrm{I}=\mathrm{I}_{0}$. For any fixed initial datum $\left(y_{0}, x_{0}\right)$, let $g_{0}:=g\left(\mathrm{I}_{0}, y_{0}, x_{0}\right)$. If $\omega\left(\mathrm{I}_{0}, g_{0}\right):=\left.\partial_{g} \tilde{h}(\mathrm{I}, g)\right|_{\left(\mathrm{I}_{0}, g_{0}\right)} \neq 0$, the motion $\left(y^{h}(t), x^{h}(t)\right)$ with initial datum $\left(y_{0}, x_{0}\right)$ under $h$ is related to the corresponding motion $\left(y^{g}(t), x^{g}(t)\right)$ under $g$ via

$$
y^{h}(t)=y^{g}\left(\omega\left(\mathrm{I}_{0}, g_{0}\right) t\right), \quad x^{h}(t)=x^{g}\left(\omega\left(\mathrm{I}_{0}, g_{0}\right) t\right) .
$$

In particular, under this condition, all the fixed points of $g$ in the plane $(y, x)$ are fixed point to $h$. Values of $\left(\mathrm{I}_{0}, g_{0}\right)$ for which $\omega\left(\mathrm{I}_{0}, g_{0}\right)=0$ provide, in the plane $(y, x)$, curves of fixed points for $h$ (which are not necessarily curves of fixed points to $g$ ).

Proof All the assertions follow from the formulae, implied by (26):

$$
\dot{y}^{h}=-h_{x}=-\tilde{h}_{x}=-\omega\left(\mathrm{I}_{0}, g_{0}\right) g_{x}\left(\mathrm{I}_{0}, y^{h}, x^{h}\right)
$$

and, similarly,

$$
\dot{x}^{h}=\omega\left(\mathrm{I}_{0}, g_{0}\right) g_{y}\left(\mathrm{I}_{0}, y^{h}, x^{h}\right)
$$

Below, we prove that, under an additional condition, the converse of Proposition 4 holds true.

Theorem 4 Let $h, g$ two commuting functions of the form (26) on the possibly complex domain $\mathcal{D}$ as in (27), with $\mathrm{I}_{i}$ pairwise Poisson commuting. For any fixed $c=\left(c_{1}, \ldots, c_{n}\right) \in \mathrm{I}(\mathcal{B})$, let $\Delta_{c}$ be the set of stationary points of the function $(y, x) \rightarrow g\left(y, x, c_{1}, \ldots, c_{n}\right)$, and put $U_{c}^{*}:=U \backslash \Delta_{c}$. Assume that the set $\mathcal{D}^{*}:=\bigcup_{(p, q) \in \mathcal{B}}\{(p, q)\} \times U_{\mathrm{I}(p, q)}^{*}$ has full closure. Then $h$ is renormalizably integrable via $g$.

Proof We firstly observe that, since $\{h, g\}=\left\{\mathrm{I}_{i}, \mathrm{I}_{j}\right\}=0$ for all $1 \leq i<j \leq n$, using, as in the proof of Proposition 4, Eq. (30), then

$$
\partial_{y} h \partial_{x} g-\partial_{y} g \partial_{x} h=\partial_{y} \widehat{h} \partial_{x} \widehat{g}-\partial_{y} \widehat{g} \partial_{x} \widehat{h}=0 .
$$

The assumptions and the implicit function theorem ensure that for any given $c=$ $\left(c_{1}, \ldots, c_{n}\right) \in \mathbb{R}^{n}$ in the image of the function $(p, q) \in \mathcal{B} \rightarrow\left(\mathrm{I}_{1}, \ldots, \mathrm{I}_{n}\right)$, and $c_{n+1}$ sufficiently close to in the image of $g\left(c_{1}, \ldots, c_{n}, \bar{y}, \bar{x}\right)$ where $(\bar{y}, \bar{x}) \in U_{c}^{*}$, equation

$$
g\left(c_{1}, \ldots, c_{n}, y, x\right)=c_{n+1}
$$

can be uniquely solved with respect to either $y$ or $x$, via suitable functions

$$
y=\mathrm{Y}\left(c_{1}, \ldots, c_{n+1}, x\right) \text { or } x=\mathrm{X}\left(c_{1}, \ldots, c_{n+1}, y\right),
$$

where $\mathrm{Y}\left(c_{1}, \ldots, c_{n+1}, \cdot\right)$ is defined on a small neighbourhood of $\bar{x}$, while $\mathrm{X}\left(c_{1}, \ldots, c_{n+1}, \cdot\right)$ is defined on a small neighbourhood of $\bar{y}$. We now consider the function

$$
\mathrm{h}\left(c_{1} \ldots c_{n}, c_{n+1}\right):=\widehat{h}\left(c_{1}, \ldots, c_{n}, \mathrm{Y}\left(c_{1}, \ldots, c_{n+1}, x\right), x\right)
$$

and/or the function

$$
\mathrm{h}^{\prime}\left(c_{1} \ldots c_{n}, c_{n+1}\right):=\widehat{h}\left(c_{1}, \ldots, c_{n}, y, \mathrm{X}\left(c_{1}, \ldots, c_{n+1}, y\right)\right) .
$$


We have that $\mathrm{h}$ is $x$-independent, while $\mathrm{h}^{\prime}$ is $y$-independent. Let us check the assertion for $\mathrm{h}$ (for $\mathrm{h}^{\prime}$ is specular). Again by the implicit function theorem:

$$
\begin{aligned}
\mathrm{h}_{x}= & \widehat{h}_{y}\left(c_{1}, \ldots, c_{n}, \mathrm{Y}\left(c_{1}, \ldots, c_{n+1}, x\right), x\right) \mathrm{Y}_{x}\left(c_{1}, \ldots, c_{n+1}, x\right) \\
& +\widehat{h}_{x}\left(c_{1}, \ldots, c_{n}, \mathrm{Y}\left(c_{1}, \ldots, c_{n+1}, x\right), x\right) \\
= & -\widehat{h}_{y}\left(c_{1}, \ldots, c_{n}, \mathrm{Y}\left(c_{1}, \ldots, c_{n+1}, x\right), x\right) \frac{\widehat{g}_{x}\left(c_{1}, \ldots, c_{n}, \mathrm{Y}\left(c_{1}, \ldots, c_{n+1}, x\right), x\right)}{\widehat{g}_{y}\left(c_{1}, \ldots, c_{n}, \mathrm{Y}\left(c_{1}, \ldots, c_{n+1}, x\right), x\right)} \\
& +\widehat{h}_{x}\left(c_{1}, \ldots, c_{n}, \mathrm{Y}\left(c_{1}, \ldots, c_{n+1}, x\right), x\right) \\
\equiv & 0
\end{aligned}
$$

because of (31). Choosing, for a fixed $(p, q) \in \mathcal{B},(y, x) \in U_{\mathrm{I}(p, q)}^{*}, c_{1}=\mathrm{I}_{1}(p, q), \ldots$, $c_{n}=\mathrm{I}_{n}(p, q), c_{n+1}=g(p, q, y, x)$, we have the thesis on the set $\mathcal{D}^{*}$. Then, by smooth continuation, the thesis holds on all of $\mathcal{D}=\mathcal{B} \times U$.

Remark 3 We observe that the proof is constructive: it provides the function $\widehat{h}$ via formulae (32)-(33).

In the following, we prove that $h_{2}$ is renormalizably integrable via $\mathrm{E}_{0}$ as an application of Theorem 4. Afterwards, in Sect. 3.1, we exhibit, explicitly, the relative function $\widetilde{h}_{2}$ realizing (29). In Sect. 3.2, as a counter-example to the last assertion of Proposition 5, we exhibit a curve of fixed points for $h_{2}$ which is not so for $\mathrm{E}_{0}$.

Application of Theorem 4 to $h_{2}$ and $\mathrm{E}_{0}$. We aim to apply Theorem 4 to $h_{2}$ and $\mathrm{E}_{0}$. As in the former section, we use the coordinates $\mathcal{K}$ defined in (15). This map turns to be useful, because the integrals $\mathrm{I}_{1}, \ldots, \mathrm{I}_{5}$ are coordinates of such system and hence depend on $(p, q)$ only via one of the $p$ 's or one of the $q$ 's: see (18). As a first step, we aim to check that $h_{2}$ and $\mathrm{E}_{0}$ have the form in (26), with

$$
n=3, \quad \mathrm{I}=\left(\mathrm{I}_{1}, \mathrm{I}_{2}, \mathrm{I}_{3}\right)=\left(\mathrm{r}_{1}, \Lambda_{2}, \Theta\right), \quad y=\mathrm{G}_{2}, \quad x=\mathrm{g}_{2} .
$$

The expression of $\mathrm{E}_{0}$ is given in (16), so it turns to be as claimed. The expression of $h_{2}$ in terms of $\mathcal{K}$ has been discussed by Pinzari (2018a) and is

$$
\begin{aligned}
& h_{2}\left(\mathrm{r}_{1}, \Lambda_{2}, \Theta, \mathrm{G}_{2}, \mathrm{~g}_{2}\right)= \frac{1}{2 \pi} \\
& \int_{\mathbb{T}} \frac{d \mathrm{l}_{2}}{\sqrt{\mathrm{r}_{1}^{2}+2 \mathrm{r}_{1} a_{2} \varrho_{2} \sqrt{1-\frac{\Theta^{2}}{\mathrm{G}_{2}^{2}}} \cos \left(\mathrm{g}_{2}+\mathrm{f}_{2}\right)+a_{2}^{2} \varrho_{2}^{2}}},
\end{aligned}
$$

where

$$
\varrho_{2}=\left(1-\sqrt{1-\frac{\mathrm{G}_{2}^{2}}{\Lambda_{2}^{2}}} \cos \zeta_{2}\right),
$$

with $\zeta_{2}$, as above, the eccentric anomaly, and $\mathrm{f}_{2}$, the true anomaly, both depending on $\left(\Lambda_{2}, \mathrm{G}_{2}, \mathrm{l}_{2}\right)$. We observe that it is possible to have a closed formula for $h_{2}$, since the integration in $d \mathrm{l}_{2}$ can be written explicitly by means of the eccentric anomaly

$$
d \mathrm{l}_{2}=\varrho_{2} d \zeta_{2}
$$

and the true anomaly $f_{2}$ can be eliminated via the well-known relation

$$
\varrho_{2} \cos \left(g_{2}+f_{2}\right)=\cos g_{2}\left(\cos \zeta_{2}-\sqrt{1-\frac{G_{2}^{2}}{\Lambda_{2}^{2}}}\right)-\frac{G_{2}}{\Lambda_{2}} \sin g_{2} \sin \zeta_{2} .
$$


Then we rewrite $h_{2}$ as

$$
\begin{aligned}
& h_{2}\left(\mathrm{r}_{1}, \Lambda_{2}, \Theta, \mathrm{G}_{2}, \mathrm{~g}_{2}\right)=\frac{1}{2 \pi} \int_{\mathbb{T}} \\
& \sqrt{\mathrm{r}_{1}^{2}+2 \mathrm{r}_{1} a_{2} \sqrt{1-\frac{\Theta^{2}}{\mathrm{G}_{2}^{2}}}\left(\cos \mathrm{g}_{2}\left(\cos \zeta_{2}-\sqrt{1-\frac{\mathrm{G}_{2}^{2}}{\Lambda_{2}^{2}}}\right)-\frac{\mathrm{G}_{2}}{\Lambda_{2}} \sin \mathrm{g}_{2} \sin \zeta_{2}\right)+a_{2}^{2} \varrho_{2}^{2}}
\end{aligned}
$$

which is precisely of the form (26). As a second step, we check that, for any fixed value of the integrals $I$ in (34), the set of fixed points of $E_{0}$ as a function of $\left(G_{2}, g_{2}\right)$ is at most one-dimensional in the plane $\left(\mathrm{g}_{2}, \mathrm{G}_{2}\right)$. Indeed, equations

$$
\left\{\begin{array}{l}
\partial_{\mathrm{G}_{2}} \mathrm{E}_{0}=0 \\
\partial_{\mathrm{g}_{2}} \mathrm{E}_{0}=0
\end{array}\right.
$$

which read

$$
\left\{\begin{array}{l}
2 \mathrm{G}_{2}\left(1-\frac{\mathrm{m}_{2}^{2} \mathrm{M}_{2} \mathrm{r}_{1}}{2 \Lambda_{2}^{2}} \frac{\sqrt{1-\frac{\Theta^{2}}{\mathrm{G}_{2}^{2}}}}{\sqrt{1-\frac{\mathrm{G}_{2}^{2}}{\Lambda_{2}^{2}}}} \cos \mathrm{g}_{2}+\frac{\mathrm{m}_{2}^{2} \mathrm{M}_{2} \mathrm{r}_{1} \Theta^{2}}{2 \mathrm{G}_{2}^{4}} \frac{\sqrt{1-\frac{\mathrm{G}_{2}^{2}}{\Lambda_{2}^{2}}}}{\sqrt{1-\frac{\Theta^{2}}{\mathrm{G}_{2}^{2}}}} \cos \mathrm{g}_{2}\right)=0 \\
\mathrm{r}_{1} \sqrt{1-\frac{\Theta^{2}}{\mathrm{G}_{2}^{2}}} \sqrt{1-\frac{\mathrm{G}_{2}^{2}}{\Lambda_{2}^{2}}} \sin \mathrm{g}_{2}=0
\end{array},\right.
$$

define an algebraic set in having positive co-dimension. Then Theorem 4 applies and we have the following

Proposition $6 h_{2}$ is renormalizably integrable via $\mathrm{E}_{0}$. Namely, there exists a function $\widetilde{h}_{2}$ such that

$$
h_{2}\left(\mathrm{r}_{1}, \Lambda_{2}, \Theta, \mathrm{G}_{2}, \mathrm{~g}_{2}\right)=\widetilde{h}_{2}\left(\mathrm{r}_{1}, \Lambda_{2}, \Theta, \mathrm{E}_{0}\left(\mathrm{r}_{1}, \Lambda_{2}, \Theta, \mathrm{G}_{2}, \mathrm{~g}_{2}\right)\right) .
$$

In the two following sections, we discuss some insights of dynamical character, related to the renormalizable integrability of $h_{2}$.

\subsection{The explicit expression of $\tilde{h}_{2}$}

The function $\widetilde{h}_{2}$ in Proposition 6 can be written explicitly, and this is the purpose of this section. Before doing it, let us premise some algebraic consideration.

Definition 2 (The class $\mathcal{H}_{*}$ ) We call class $\mathcal{H}_{*}$ the set of functions of the form

$$
f(a, b, u, v)=\frac{1}{2 \pi} \int_{\mathbb{T}} \frac{P(u c(w)) d w}{\sqrt{a^{2}+2 a b Q(v s(w))+b^{2} P(u c(w))^{2}}},
$$

where: $u \rightarrow P(u), u \rightarrow Q(u)$ are smooth functions for $u=0 ; P(0)>0 ; u \rightarrow Q(u)$ is odd; $c, s$ are periodic functions such that there exist two "symmetries", i.e. transformations $\sigma, \sigma^{\prime}: \mathbb{T} \rightarrow \mathbb{T}$ verifying $\left|\partial_{w} \sigma\right|=\left|\partial_{w} \sigma^{\prime}\right| \equiv 1$ and

$$
c \circ \sigma=c, \quad c \circ \sigma^{\prime}=-c, \quad s \circ \sigma=-s, \quad s \circ \sigma^{\prime}=s .
$$


Definition 2 implies that any $f \in \mathcal{H}_{*}$ is homogeneous of degree -1 in $(a, b)$; even in all of their arguments

$$
\begin{aligned}
f(-a, b, u, v) & =f(a,-b, u, v)=f(a, b,-u, v)=f(a, b, u,-v) \\
& =f(a, b, u, v) \quad \forall(a, b, u, v)
\end{aligned}
$$

and, moreover, verifies

$$
f(1,0, u, v)=f(0,1, u, v)=1 \quad \forall(u, v) .
$$

Proposition 7 All the functions in $\mathcal{H}_{*}$ afford a formal series expansion

$$
f=\sum_{h, k} f_{h k}(a, b) u^{2 h} v^{2 k}
$$

with

$$
f_{h k}(a, b)=\frac{a^{2} b^{2} p_{h k}(a, b)}{q(a, b)^{\frac{1}{2}+2(h+k)}} \text { for }(i, j) \in \mathbb{N}^{2} \backslash\{(0,0)\},
$$

where $q(a, b)$ is a positive definite quadratic form and $p_{i j}(a, b)$ are polynomials of degree $4(i+j-1)$ with coefficients in $\mathbb{Q}$, even separately in a and b. In particular, for any $f \in \mathcal{H}_{*}$, there exist $r, s \in \mathbb{Q}$ such that

$$
r f_{10}(a, b)+s f_{01}(a, b) \equiv 0 \quad \forall(a, b) \in \mathbb{R}^{2} .
$$

Remark 4 We call identity (43) generalized Herman resonance and underline that its validity is strongly based on identity (40). For the averaged Newtonian potential, (40) is guaranteed by the Keplerian property (Proposition 1).

Proof Using formula (38), it is easy to prove, by induction, that any $f \in \mathcal{H}_{*}$ affords an expansion of the kind

$$
f=\sum_{i, j} \bar{f}_{i j}(a, b) u^{i} v^{j}
$$

with

$$
\bar{f}_{i j}(a, b)=\frac{\bar{p}_{i j}(a, b)}{q(a, b)^{\frac{1}{2}+i+j}},
$$

where $\bar{p}_{i j}(a, b)$ are polynomials in $(a, b)$ and

$$
q(a, b)=a^{2}+P(0)^{2} b^{2} .
$$

Using the parity of $f$ with respect to all of its arguments, one has, actually, that $\bar{p}_{i j}$ 's are even with respect to $a$ and $b$ separately, and vanish if $i, j$ are not both even, so we have an expansion of the form (41), with $f_{h k}=\bar{f}_{2 h, 2 k}$. Furthermore, since $f$ is homogeneous of degree -1 , all of its derivatives with respect to $u$ or $v$ are homogeneous of the same degree. Since $q(a, b)$ is homogeneous of degree 2 (see 45), we have that the $\bar{p}_{2 h, 2 k}$ in (44) are to be homogeneous of degree $4(h+k)$. Finally, due to $(40), \bar{p}_{2 h, 2 k}(1,0)=\bar{p}_{2 h, 2 k j}(0,1) \equiv 0$ for all $(h, k) \neq(0,0)$. Combining this with parity of $\bar{p}_{2 h, 2 k}$ with respect to $a$ and $b$ separately, (42), $\bar{p}_{2 h, 2 k}(a, b)=a^{2} b^{2} p_{h k}(a, b)$ where $p(h, k)(a, b)$ has degree $4(h+k-1)$. This proves the former assertion. The latter follows from this, since, when $h+k=1$,

$$
f_{10}=\frac{a^{2} b^{2} p_{10}(a, b)}{q(a, b)^{\frac{5}{2}}}, \quad f_{01}=\frac{a^{2} b^{2} p_{01}(a, b)}{q(a, b)^{\frac{5}{2}}},
$$


with $p_{10}$ and $p_{01}$ having degree 0 , namely, $p_{10}$ and $p_{01} \in \mathbb{Q}$. So, one can take $r=-p_{01}$, $s=p_{10}$.

Let us now proceed to write down an explicit expression of function $\widetilde{h}_{2}$ in Proposition 6. We let

$$
\begin{aligned}
\mathrm{U}(a, b, u, v) & =\frac{1}{2 \pi} \int_{\mathbb{T}} \frac{(1-u \cos w) d w}{\sqrt{a^{2}+b^{2}-2 b(a v \sin w+b u \cos w)+b^{2} u^{2} \cos ^{2} w}} \\
\mathcal{E}\left(\Lambda_{2}, \mathrm{E}_{0}\right) & =\frac{\sqrt{\Lambda_{2}^{2}-\mathrm{E}_{0}}}{\Lambda_{2}} \mathcal{I}\left(\Lambda_{2}, \Theta, \mathrm{E}_{0}\right)=\frac{\sqrt{\mathrm{E}_{0}-\Theta^{2}}}{\Lambda_{2}} .
\end{aligned}
$$

Note that $\mathrm{U}$ is in the class $\mathcal{H}_{*}$, with

$$
\begin{aligned}
P(u, v) & =1-u, \quad Q(v)=v, \quad c(w)=\cos w, \quad s(w)=\sin w, \quad \sigma(w)=-w \\
\sigma^{\prime}(w) & =\pi-w .
\end{aligned}
$$

We prove that

Proposition $8 \tilde{h}_{2}\left(\mathrm{r}_{1}, \Lambda_{2}, \Theta, \mathrm{E}_{0}\right)=\mathrm{U}\left(\mathrm{r}_{1}, a_{2}, \mathcal{E}\left(\Lambda_{2}, \mathrm{E}_{0}\right), \mathcal{I}\left(\Lambda_{2}, \Theta, \mathrm{E}_{0}\right)\right)$.

Proof Reasoning as in the proof of Theorem 4 (see Remark 3), we invert equation

$$
\mathrm{E}_{0}\left(\mathrm{r}_{1}, \Lambda_{2}, \Theta, \mathrm{G}_{2}, \mathrm{~g}_{2}\right)=\overline{\mathrm{E}}_{0}
$$

with respect to $G_{2}$ in the complex field, fixing a value of $g_{2}$. We choose $g_{2}=\frac{\pi}{2}$, so that $\cos \mathrm{g}_{2}=0$ and the inversion is immediate:

$$
\mathrm{G}_{2}=\sqrt{\overline{\mathrm{E}}_{0}} .
$$

Then $\widetilde{h}_{2}\left(\mathrm{r}_{1}, \Lambda_{2}, \Theta, \overline{\mathrm{E}}_{0}\right)$ is given by

$$
\widetilde{h}_{2}\left(\mathrm{r}_{1}, \Lambda_{2}, \Theta, \overline{\mathrm{E}}_{0}\right)=h_{2}\left(\mathrm{r}_{1}, \Lambda_{2}, \Theta, \sqrt{\overline{\mathrm{E}}_{0}}, \frac{\pi}{2}\right) \text {. }
$$

Using the formula in (36), we obtain

$$
\begin{aligned}
& \tilde{h}_{2}\left(\mathrm{r}_{1}, \Lambda_{2}, \Theta, \mathrm{E}_{0}\right)=\frac{1}{2 \pi} \int_{\mathbb{T}} d \zeta_{2} \\
& \frac{1-\mathcal{E}\left(\Lambda_{2}, \mathrm{E}_{0}\right) \cos \zeta_{2}}{\sqrt{\mathrm{r}_{1}^{2}+a_{2}^{2}-2 a_{2}\left(\mathrm{r}_{1} \mathcal{I}\left(\Lambda_{2}, \Theta, \mathrm{E}_{0}\right) \sin \zeta_{2}+a_{2} \mathcal{E}\left(\Lambda_{2}, \mathrm{E}_{0}\right) \cos \zeta_{2}\right)+a_{2}^{2} \mathcal{E}\left(\Lambda_{2}, \mathrm{E}_{0}\right)^{2} \cos ^{2} \zeta_{2}}},
\end{aligned}
$$

with $\mathcal{E}, \mathcal{I}$ as in (47).

Remark 5 Combining Propositions 6, 8 with (7) and the definitions of $\mathrm{G}_{2}$ and $\Theta$ in (15), we obtain that, for a generic $\mathcal{C}$ as in (1),

$$
h_{2}=\mathrm{U}\left(\left\|x_{\mathcal{C}}^{(1)}\right\|, a_{2}, \mathcal{E}_{\mathcal{C}}, \mathcal{I}_{\mathcal{C}}\right)
$$

with

$$
\begin{aligned}
& \mathcal{E}_{\mathcal{C}}:=\left(\sqrt{\mathrm{e}_{2}^{2}+\mathrm{e}_{2} \frac{x^{(1)} \cdot \mathrm{P}^{(2)}}{a_{2}}}\right) \circ \mathcal{C}, \\
& \mathcal{I}_{\mathcal{C}}:=\left(\sqrt{\frac{\left\|x^{(1)}\right\|^{2}\left\|\mathrm{C}^{(2)}\right\|^{2}-\left(x^{(1)} \cdot \mathrm{C}^{(2)}\right)^{2}}{\left\|x^{(1)}\right\|^{2} \Lambda_{2}^{2}}-\mathrm{e}_{2} \frac{x^{(1)} \cdot \mathrm{P}^{(2)}}{a_{2}}}\right) \circ \mathcal{C} .
\end{aligned}
$$

In Sect. 5.1, we use the following consequence of this. 


\section{Proposition 9 Let}

$$
\mathcal{C}_{2}: \quad\left(\Lambda_{2}, \ell_{2}, \bar{u}, \bar{v}\right) \in \mathcal{A} \times \mathbb{T} \times U \rightarrow\left(y^{(2)}, x^{(2)}\right) \in\left(\mathbb{R}^{3}\right)^{2},
$$

where $U$ is a domain of $\mathbb{R}^{4}$, verify (2) and let $\tilde{v} \in \mathbb{R}^{3}$. Then

$$
\begin{aligned}
& \frac{1}{2 \pi} \int_{\mathbb{T}} \frac{d \ell_{2}}{\left\|\widetilde{v}-x_{\mathcal{C}_{2}}^{(2)}\right\|}=\mathrm{U}\left(\|\tilde{v}\|, a_{2}, \mathcal{E}_{2}, \mathcal{I}_{2}\right), \\
& \mathcal{E}_{2}:=\sqrt{\mathrm{e}_{2, \mathcal{C}_{2}}^{2}+\mathrm{e}_{2, \mathcal{C}_{2}} \frac{\tilde{v} \cdot \mathrm{P}_{\mathcal{C}_{2}}^{(2)}}{a_{2}}}, \mathcal{I}_{2}:=\sqrt{\frac{\|\widetilde{v}\|^{2}\left\|\mathrm{C}_{\mathcal{C}_{2}}^{(2)}\right\|^{2}-\left(\widetilde{v} \cdot \mathrm{C}_{\mathcal{C}_{2}}^{(2)}\right)^{2}}{\|\widetilde{v}\|^{2} \Lambda_{2}^{2}}-\mathrm{e}_{2, \mathcal{C}_{2}} \frac{\tilde{v} \cdot \mathrm{P}_{\mathcal{C}_{2}}^{(2)}}{a_{2}}},
\end{aligned}
$$

where the sub-fix $\mathcal{C}_{2}$ denotes the composition with $\mathcal{C}_{2}$.

Proof Choose $\mathcal{C}=\mathrm{id} \otimes \mathcal{C}_{2}$ in (1); namely, such that $u=(\widetilde{u}, \bar{u}), v=(\widetilde{v}, \bar{v}) \in \mathbb{R}^{3} \times \mathbb{R}^{2}$, with $\left(y^{(1)}, x^{(1)}\right) \circ \mathcal{C}=(\widetilde{u}, \widetilde{v}) \in \mathbb{R}^{3} \times \mathbb{R}^{2}$, and $\left(y^{(2)}, x^{(2)}\right) \circ \mathcal{C}=\left(y^{(2)}, x^{(2)}\right) \circ \mathcal{C}_{2}$, depending only on $\left(\Lambda_{2}, \ell_{2}, \bar{u}, \bar{v}\right)$.

\subsection{A curve of fixed points for $h_{2}$ (which is not so for $E_{0}$ )}

Proposition 6 implies that any level set (in the plane $(\mathrm{G}, \mathrm{g})$ ) to $\mathrm{E}_{0}$ is also a level set of $h_{2}$ and hence, in particular, any fixed point to $\mathrm{E}_{0}$ is so to $h_{2}$. Here, we prove that the converse is not true:

Proposition 10 If $\Theta \neq 0$ and $\mathrm{r}_{1} / a_{2}$ is sufficiently small, in the plane $\left(\mathrm{G}_{2}, \mathrm{~g}_{2}\right)$, there exists at least a curve of fixed points of $h_{2}$ which is a level set of $\mathrm{E}_{0}$, but is not a curve a fixed points to it.

Proof In principle, to find any such curve, one should solve equation $\omega(\mathrm{I}, \tilde{h}):=\partial_{g} \tilde{h}(\mathrm{I}, \tilde{h})=$ 0 . In the case of $h_{2}$, such equation seems too difficult, so we shall use a perturbative approach. We look at the Taylor expansion (20) of $h_{2}$ in (35) in powers of $r_{1}$. Letting $\varepsilon:=\frac{r_{1}}{a_{2}}$, we obtain

$$
\begin{aligned}
h_{2}= & \frac{1}{a_{2}}\left[1-\frac{\varepsilon^{2}}{4} \frac{\Lambda_{2}^{3}\left(3 \Theta^{2}-\mathrm{G}_{2}^{2}\right)}{\mathrm{G}_{2}^{5}}-\frac{3}{8} \varepsilon^{3} \sqrt{1-\frac{\mathrm{G}_{2}^{2}}{\Lambda_{2}^{2}}} \sqrt{1-\frac{\Theta^{2}}{\mathrm{G}_{2}^{2}}} \frac{\Lambda_{2}^{5}}{\mathrm{G}_{2}^{5}}\left(1-5 \frac{\Theta^{2}}{\mathrm{G}_{2}^{2}}\right) \cos \mathrm{g}_{2}\right. \\
& \left.+\mathrm{O}\left(\varepsilon^{4}\right)\right] .
\end{aligned}
$$

By (48), a corresponding expansion for the function $\widetilde{h}$ in (49) is obtained letting $\mathrm{G}_{2}=\sqrt{\mathrm{E}_{0}}$ and $g_{2}=\frac{\pi}{2}$. We obtain:

$$
\tilde{h}_{2}\left(\mathrm{r}_{1}, \Lambda_{2}, \Theta, \mathrm{E}_{0}\right)=\frac{1}{a_{2}}\left[1-\frac{\varepsilon^{2}}{4} \frac{\Lambda_{2}^{3}\left(3 \Theta^{2}-\mathrm{E}_{0}\right)}{\mathrm{E}_{0}^{5 / 2}}+\mathrm{O}\left(\varepsilon^{4}\right)\right] .
$$

We study equation

$$
\tilde{\omega}=\partial_{\mathrm{E}_{0}} \tilde{h}=-\frac{\varepsilon^{2}}{a_{2}}\left[\frac{\Lambda_{2}^{3}}{4} \frac{-15 \Theta^{2}+3 \mathrm{E}_{0}}{\mathrm{E}_{0}^{7 / 2}}+\mathrm{O}\left(\varepsilon^{2}\right)\right]=0
$$

via the implicit function theorem, for small $\varepsilon$. Neglecting the $\mathrm{O}\left(\varepsilon^{2}\right)$ inside parentheses, we obtain the solution

$$
\mathrm{E}_{0}=5 \Theta^{2}
$$


The non-degeneracy condition at this solution is verified, since indeed

$$
\left.\partial_{\mathrm{E}_{0}} \frac{-15 \Theta^{2}+3 \mathrm{E}_{0}}{\mathrm{E}_{0}^{7 / 2}}\right|_{\mathrm{E}_{0}=5 \Theta^{2}}=15 \frac{7}{2 \Theta^{7}}-3 \frac{5}{2 \Theta^{7}}=\frac{45}{\Theta^{2}} \neq 0 .
$$

Then for sufficiently small $\varepsilon$, Eq. (51) has, as a solution, the following level set of $\mathrm{E}_{0}$ :

$$
\mathrm{E}_{0}=5 \Theta^{2}+\mathrm{O}\left(\varepsilon^{2}\right)
$$

Replacing the formula for $\mathrm{E}_{0}$ in (16), we rewrite such solution as the curve, in the $\left(\mathrm{G}_{2}, \mathrm{~g}_{2}\right)$ plane,

$$
\mathcal{S}: \quad \mathrm{G}_{2}^{2}-5 \Theta^{2}+\mathrm{m}_{2}^{2} \mathrm{M}_{2} \mathrm{r}_{1} \sqrt{1-\frac{\mathrm{G}_{2}^{2}}{\Lambda_{2}^{2}}} \sqrt{1-\frac{\Theta^{2}}{\mathrm{G}_{2}^{2}}} \cos \mathrm{g}_{2}+\mathrm{O}\left(\mathrm{r}_{1}^{2}\right)=0 .
$$

By Proposition 5, $\mathcal{S}$ is a curve of fixed points for $h_{2}$. It remains to prove that $\mathcal{S}$ is not a curve of fixed points for $\mathrm{E}_{0}$. The fixed points of $\mathrm{E}_{0}$ are the solutions of system (37). The curve $\mathcal{S}$ includes a point having coordinates

$$
\mathrm{G}_{2}=\sqrt{5} \Theta+\mathrm{O}\left(\mathrm{r}_{1}^{2}\right), \quad \mathrm{g}_{2}=\frac{\beta}{2}+\mathrm{O}\left(\mathrm{r}_{1}\right)
$$

which does not solve system (37). (It does not solve the second equation.)

Remark 6 The proof fails for $\Theta=0$, because, in such a case, the leading part in Eq. (51) has no solution.

\section{An algebraic property of Legendre polynomials}

The Legendre polynomials $\mathcal{P}_{n}(t)$, with $\mathcal{P}_{0}(t)=1, \mathcal{P}_{1}(t)=t, \ldots$, are defined via the $\varepsilon$ expansion

$$
\frac{1}{\sqrt{1-2 \varepsilon t+\varepsilon^{2}}}=\sum_{n=0}^{\infty} \mathcal{P}_{n}(t) \varepsilon^{n} .
$$

Many notices on such classical polynomials may be found in Giorgilli (2008, Appendix B).

The purpose of this section is to present an algebraic property of the $\mathcal{P}_{n}$ 's. Roughly, it says that a certain average of a Legendre polynomial is still a Legendre polynomial. The author is not aware if it was known before and if there is a "dynamical" explanation of it.

Lemma 2 Let $t \in \mathbb{R},|t|<1, \mathcal{P}_{n}$ the nth Legendre polynomial. Then,

$$
\frac{1}{2 \pi} \int_{\mathbb{T}} \mathcal{P}_{n}\left(\sqrt{1-t^{2}} \cos \theta\right) d \theta=\delta_{n} \mathcal{P}_{n}(t)
$$

where

$$
\delta_{n}= \begin{cases}(-1)^{m} \frac{(2 m-1) ! !}{(2 m) ! !} & \text { if } n=2 m \text { is even } \\ 0 & \text { if } n \text { is odd }\end{cases}
$$

We shall prove Lemma 2 via the following one. 
Lemma 3 The even Legendre polynomials $P_{2 m}(t)$ verify, for any $h=0, \ldots, m$,

$$
\begin{aligned}
& D_{\tau}^{h} P_{2 m}(0)=(-1)^{m-h} \frac{h !}{(2 h) !} \frac{(2 m-2 h-1) ! !}{(2 m-2 h) ! !}, \\
& D_{\tau}^{h} P_{2 m}(1)=\frac{1}{2^{h}} \frac{(2 m+2 h-1) ! !}{(2 m-1) ! !} \frac{(2 m) ! !}{(2 h) ! !(2 m-2 h) ! !},
\end{aligned}
$$

where $\tau:=t^{2}$. In particular, the following relation holds

$$
(-1)^{h} \frac{(2 h-1) ! !}{(2 h) ! !} D_{\tau}^{h} P_{2 m}(0)=(-1)^{m} \frac{(2 m-1) ! !}{(2 m) ! !} D_{\tau}^{h} P_{2 m}(1) .
$$

Proof We first prove the former formula in (53). Let $n \in \mathbb{N}, k=0, \ldots, n$ with $n-k$ even. We have $\mathrm{x}$

$$
\left.D_{t}^{k} \frac{1}{\sqrt{\varepsilon^{2}-2 t \varepsilon+1}}\right|_{t=0}=(2 k-1) ! ! \frac{\varepsilon^{k}}{\left(1+\varepsilon^{2}\right)^{\frac{2 k+1}{2}}} .
$$

Therefore, denoting as $\Pi_{n}$ the projection over the monomial $\varepsilon^{n}$,

$$
\begin{aligned}
D_{t}^{k} \mathcal{P}_{n}(0) & =\left.D_{t}^{k}\left(\Pi_{n} \frac{1}{\sqrt{\varepsilon^{2}-2 t \varepsilon+1}}\right)\right|_{t=0}=\Pi_{n}\left(\left.D_{t}^{k} \frac{1}{\sqrt{\varepsilon^{2}-2 t \varepsilon+1}}\right|_{t=0}\right) \\
& =(2 k-1) ! ! \Pi_{n-k} \frac{1}{\left(1+\varepsilon^{2}\right)^{\frac{2 k+1}{2}}} \\
& =\left.\frac{(2 k-1) ! !}{((n-k) / 2) !} D_{\eta}^{(n-k) / 2} \frac{1}{(1+\eta)^{\frac{2 k+1}{2}}}\right|_{\eta=0}=(-1)^{(n-k) / 2} \frac{(k+n-1) ! !}{2^{(n-k) / 2}((n-k) / 2) !} \\
& =(-1)^{(n-k) / 2} \frac{(k+n-1) ! !}{(n-k) ! !} .
\end{aligned}
$$

Then the desired formula follows, taking $n=2 m, k=2 h$ and noticing that

$$
D_{\tau}^{h} P_{2 m}(0)=\frac{h !}{(2 h) !} D_{t}^{2 h} P_{2 m}(0) .
$$

The proof of the latter formula in (53) is a bit more complicate. We propose an algebraic one.

First of all, we change variable

$$
t=\sqrt{\tau}=\sqrt{1-2 z} .
$$

Since

$$
D_{\tau}^{h}=\frac{(-1)^{h}}{2^{h}} D_{z}^{h},
$$

we are definitely reduced to prove the following identity

$$
\begin{aligned}
\left.D_{z}^{h} P_{2 m}(\sqrt{1-2 z})\right|_{z=0}= & D_{2 m, 2 h}:=\frac{(-1)^{h}}{(2 h) !}(2 m-2 h+2)(2 m-2 h+4) \cdots(2 m) \\
& \times(2 m+1)(2 m+3) \cdots(2 m+2 h-1)
\end{aligned}
$$

To this end, we let

$$
g(\varepsilon, z):=\frac{1}{\sqrt{\varepsilon^{2}-2 \varepsilon \sqrt{1-2 z}+1}},
$$


so that (analogously to (54)) we may identify

$$
\left.D_{z}^{h} P_{2 m}(\sqrt{1-2 z})\right|_{z=0}=\left.\Pi_{2 m} D_{z}^{h} g(\varepsilon, z)\right|_{z=0} .
$$

We introduce the auxiliary functions

$$
g_{a, b}(\varepsilon, z)=\frac{1}{\left(\varepsilon^{2}-2 \varepsilon \sqrt{1-2 z}+1\right)^{\alpha / 2}} \frac{1}{(1-2 z)^{\beta / 2}} \quad \alpha, \beta \in \mathbb{R}
$$

so that $g_{1,0}=g$. Observe that the linear space generated by such functions is closed under the derivative operation, since in fact

$$
D_{z} g_{a, b}(\varepsilon, z)=-\varepsilon \alpha g_{\alpha+2, \beta+1}(\varepsilon, z)+\beta g_{a, b+2}(\varepsilon, z) .
$$

More in general, by iteration, one finds

$$
D_{z}^{h} g_{a, b}(\varepsilon, z)=\sum_{j=0}^{h} c_{j}^{(h)} \varepsilon^{j} g_{\alpha+2 j, \beta+2 h-j}(\varepsilon, z),
$$

where, from the identity

$$
D_{z}^{h+1} g_{a, b}(\varepsilon, z)=D_{z}\left(D_{z}^{h} g_{a, b}\right)(\varepsilon, z),
$$

one easily sees that the coefficients $c_{j}^{(h)}$, with $j=0, \ldots, h$, satisfy the recursion

$$
\left\{\begin{array}{l}
c_{0}^{(0)}=1 \\
c_{j}^{(h+1)}=-c_{j-1}^{(h)}(\alpha+2 j-2)+(\beta+2 h-j) c_{j}^{(h)} \\
h=0,1, \ldots ; \quad j=0,1, \ldots h+1 \\
c_{-1}^{(h)}:=0, \quad c_{h+1}^{(h)}:=0
\end{array}\right.
$$

Let $\bar{c}_{j}^{(h)}$, s be the numbers defined by

$$
\left\{\begin{array}{l}
\bar{c}_{0}^{(0)}=1 \\
\bar{c}_{j}^{(h+1)}=-\bar{c}_{j-1}^{(h)}(2 j-1)+(2 h-j) \bar{c}_{j}^{(h)} \\
h=0,1, \ldots ; \quad j=0,1, \ldots h+1 \\
\bar{c}_{-1}^{(h)}:=0, \quad \bar{c}_{h+1}^{(h)}:=0
\end{array}\right.
$$

corresponding to the case

$$
\alpha=1, \quad \beta=0 .
$$

Specializing formula (57) to this case, we find

$$
\begin{aligned}
\left.D_{z}^{h} g(\varepsilon, z)\right|_{z=0} & =\left.D_{z}^{h} g_{1,0}(\varepsilon, z)\right|_{z=0} \\
& =\left.\sum_{j=0}^{h} \bar{c}_{j}^{(h)} \varepsilon^{j} g_{1+2 j, 2 h-j}(\varepsilon, z)\right|_{z=0}=\sum_{j=0}^{h} \bar{c}_{j}^{(h)} \frac{\varepsilon^{j}}{(1-\varepsilon)^{1+2 j}} .
\end{aligned}
$$


Therefore, applying (56), we find the desired derivatives

$$
\left.D_{z}^{h} P_{2 m}(\sqrt{1-2 z})\right|_{z=0}=\sum_{j=0}^{h} C_{2 m, j} \bar{c}_{j}^{(h)},
$$

with

$$
C_{2 m, j}:=\frac{(2 m-j+1)(2 m-j+2) \cdots(2 m+j)}{(2 j) !} .
$$

In order to check (55), let $\mathcal{P}_{2 h}(\mu), \mathcal{Q}_{2 j}(\mu)$ the polynomials in the real variable $\mu$ defined as the extensions of $D_{2 m, 2 h}, C_{2 m, 2 h}$ on the reals, i.e. such that

$$
\mathcal{P}_{2 h}(2 m)=D_{2 m, 2 h}, \quad \mathcal{Q}_{2 j}(2 m)=C_{2 m, 2 j}
$$

and let

$$
\mathcal{D}_{2 h}(\mu):=\sum_{j=0}^{h} \bar{c}_{j}^{(h)} \mathcal{Q}_{2 j}(\mu)
$$

the analogous polynomial extending the right-hand side of (59). We shall prove that

$$
\mathcal{D}_{2 h}(\mu)=\mathcal{P}_{2 h}(\mu) \quad \forall \mu \in \mathbb{R}, \quad h=0,1, \ldots,
$$

which clearly implies (55). Note that $\mathcal{D}_{2 h}(\mu), \mathcal{P}_{2 h}(\mu)$ have degree $2 h ; \mathcal{P}_{2 h}(\mu)$ vanishes at the odd integers $-(2 h-1),-(2 h-3), \ldots,-1$, and the even integers $0,2, \ldots, 2 h-2$, while the $\mathcal{Q}_{2 j}(\mu)$ 's have degree $2 j$ and vanish at the integers $-j,-j+1, \ldots, j-1$. The last formula in (60) provides a decomposition of $\mathcal{D}_{2 h}(\mu)$ on the basis of the $\mathcal{Q}_{2 j}$ 's. We then do the same for $\mathcal{P}_{2 h}$, i.e. we decompose

$$
\mathcal{P}_{2 h}=\sum_{j=0}^{h} \hat{c}_{j}^{(h)} \mathcal{Q}_{2 j} .
$$

We now need to show that

$$
\hat{c}_{j}^{(h)}=\bar{c}_{j}^{(h)} \forall h=0,1, \ldots ; \quad j=0,1, \ldots, h .
$$

From the relations

$$
\mathcal{P}_{2 h+2}(\mu)=-\frac{(\mu-2 h)(\mu+2 h+1)}{2 h+2} \mathcal{P}_{2 h}(\mu)
$$

and

$$
-(\mu-2 h)(\mu+2 h+1)=(2 h-j)(2 h+j+1)-(\mu-j)(\mu+j+1),
$$

the following recursion rule among the coefficients immediately follows

$$
\left\{\begin{array}{l}
\hat{c}_{0}^{(0)}=1 \\
\hat{c}_{j}^{(h+1)}=-\frac{j(2 j-1)}{h+1} \hat{c}_{j-1}^{(h)}+\frac{4 h^{2}-j^{2}+2 h-j}{2 h+2} \hat{c}_{j}^{(h)} \\
h=0,1, \ldots ; \quad j=0,1, \ldots h+1 \\
\hat{c}_{-1}^{(h)}:=0, \quad \bar{c}_{h+1}^{(h)}:=0
\end{array} .\right.
$$


Let

$$
\delta_{j}^{(h)}:=\hat{c}_{j}^{(h)}-\bar{c}_{j}^{(h)} .
$$

The formulae in (58) and (62) imply

$$
\left\{\begin{array}{l}
\delta_{0}^{(0)}=0 \\
\delta_{j}^{(h+1)}=-\frac{(2 j+1)(j-h-1)}{h+1} \delta_{j-1}^{(h)}+\frac{(2 h-j)(j-1)}{2(h+1)} \delta_{j}^{(h)} \\
h=0,1, \ldots ; \quad j=0,1, \ldots h+1 \\
\delta_{-1}^{(h)}:=0, \quad \delta_{h+1}^{(h)}:=0
\end{array} .\right.
$$

Those relations immediately enforce, by induction, $\delta_{j}^{(h)} \equiv 0$ for all $h, j$, and hence (61).

Proof of Lemma 2 Let $\mathcal{Q}_{n}(t)$ denote the left-hand side of (52). Observe that, since any $\mathcal{P}_{n}(t)$ has the same parity, in $t$, as $n$ and odd powers of $\cos \theta$ have vanishing average, the $\mathcal{Q}_{2 m+1}(t)$ 's vanish, while the $\mathcal{Q}_{2 m}(t)$ 's are polynomials of degree $m$ in $\tau:=t^{2}$. Since also the even Legendre polynomials $\mathcal{P}_{2 m}$ 's are polynomial of degree $m$ in $\tau$, we only need to show, e.g. that

$$
\left.D_{\tau}^{h} Q_{2 m}\right|_{\tau=1}=\left.(-1)^{m} \frac{(2 m-1) ! !}{(2 m) ! !} D_{\tau}^{h} P_{2 m}\right|_{\tau=1} \quad \forall h=0, \ldots, m .
$$

The definition of $Q_{2 m}$ implies that, for $h=1, \ldots, m$

$$
D_{\tau}^{h} Q_{2 m}(1)=(-1)^{h} \overline{(\cos \theta)^{2 h}} D_{\tau}^{h} P_{2 m}(0)=(-1)^{h} \frac{(2 h-1) ! !}{(2 h) ! !} D_{\tau}^{h} P_{2 m}(0) \quad h=0, \ldots, m,
$$

where

$$
\overline{(\cos \theta)^{2 h}}:=\frac{1}{2 \pi} \int_{0}^{2 \pi}(\cos \theta)^{2 h} d \theta=\frac{(2 h-1) ! !}{(2 h) ! !} .
$$

Using Lemma 3, we find

$$
D_{\tau}^{h} Q_{2 m}(1)=(-1)^{m} \frac{(2 m-1) ! !}{(2 m) ! !} D_{\tau}^{h} P_{2 m}(1) \quad h=0, \ldots, m
$$

and hence the thesis follows.

\section{Applications}

\subsection{An explicit formula for a semi-axes-eccentricities-inclination expansion of a "mixed" averaged Newtonian potential.}

In this section, we assume that the map $\mathcal{C}$ in (1) satisfies the following conditions:

- the coordinates $(u, v)$ include

$$
u_{1}:=\Lambda_{1}, \quad v_{1}:=\ell_{1} \in \mathbb{T}, \quad v_{2}:=\mathrm{g}_{1} \in \mathbb{T},
$$


where, in addition to (2), also the following holds

$$
\left(\frac{\left\|y^{(1)}\right\|^{2}}{2 \mathrm{~m}_{1}}-\frac{\mathrm{m}_{1} \mathrm{M}_{1}}{\left\|x^{(1)}\right\|}\right) \circ \mathcal{C}=-\frac{\mathrm{m}_{1}^{3} \mathrm{M}_{1}^{2}}{2 \Lambda_{1}^{2}}=: \mathrm{h}_{\mathrm{Kep}}^{(1)}\left(\Lambda_{1}\right),
$$

with suitable other mass parameters $\mathrm{m}_{1}, \mathrm{M}_{1} ; \ell_{1}$ in conjugate to $\Lambda_{1}$;

- the image of $\mathcal{C}$ in (1) is a domain of $(y, x)$ where the left-hand side of (64) takes negative values;

- the instantaneous ellipse $\mathbb{E}_{1}$ generated by the two-body Hamiltonian (64) has nonvanishing eccentricity;

- if $\mathrm{P}^{(1)},\left\|\mathrm{P}^{(1)}\right\|=1$ denotes the direction of its perihelion, and, as above, $\mathrm{C}^{(1)}:=x^{(1)} \times$ $y^{(1)}$, the angle $\mathrm{g}_{1}$ in (63) corresponds to the anomaly of $\mathrm{P}^{(1)}$ with respect to a prefixed direction $v_{1}$ in (and a prefixed orientation of) the plane orthogonal to $\mathrm{C}^{(1)}$;

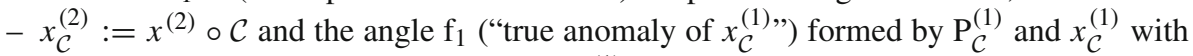
respect to the orientation established by $\mathrm{C}_{\mathcal{C}}^{(1)}$ do not depend on $\mathrm{g}_{1}$;

- if $\mathcal{D}_{i}=\left(\Lambda_{i}, 1_{i}, p_{i}, q_{i}\right)$, with $p_{i}=\left(p_{i 1}, p_{i 2}\right) \in \mathbb{R}^{2}, q_{i}=\left(q_{i 1}, q_{i 2}\right) \in \mathbb{R}^{2}$ are the Delaunay coordinates associated with $\left(y^{(i)}, x^{(i)}\right)$, and $\mathcal{D}:=\mathcal{D}_{1} \otimes \mathcal{D}_{2}:=(\Lambda, 1, p, q):=$ $\left(\Lambda_{1}, \Lambda_{2}, 1_{1}, 1_{2}, p_{11}, p_{12}, p_{21}, p_{22}, q_{11}, q_{12}, q_{21}, q_{22}\right)$, the change in coordinates

$$
\phi_{\mathcal{C}}^{\mathcal{D}}: \quad \mathcal{D}_{1} \otimes \mathcal{D}_{2} \rightarrow \mathcal{C}
$$

has the form

$$
\phi_{\mathcal{C}}^{\mathcal{D}}: \quad \ell_{2}=1_{2}+\varphi_{2}\left(\Lambda, 1_{1}, p, q\right), \quad\left(\Lambda, \ell_{1}, u, v\right)=\mathcal{F}\left(\Lambda, 1_{1}, p, q\right) .
$$

Our purpose is to provide, under the previous assumptions, a representation formula for the function $^{8}$

$$
h_{12}:=\frac{1}{(2 \pi)^{2}} \int_{\mathbb{T}^{2}} \frac{d \mathrm{~g}_{1} d \ell_{2}}{\left\|x_{\mathcal{C}}^{(1)}-x_{\mathcal{C}}^{(2)}\right\|}
$$

which we believe may turn to be useful in applications. We introduce the following

Definition 3 For a given power series in the parameter $\varepsilon$

$$
g_{\varepsilon}:=\sum_{n=0}^{\infty} a_{n} \varepsilon^{n}
$$

we denote as $\Pi_{\varepsilon} g_{\varepsilon}$ the even power series

$$
\Pi_{\varepsilon} g_{\varepsilon}:=\sum_{m=0}^{\infty}(-1)^{m} \frac{(2 m-1) ! !}{(2 m) ! !} a_{2 m} \varepsilon^{2 m},
$$

with $(-1) ! !:=1$.

8 The reader should not confuse the function $h_{12}$ above with what is commonly called "doubly averaged Newtonian potential", defined as

$$
\bar{h}_{12}:=\frac{1}{(2 \pi)^{2}} \int_{\mathbb{T}^{2}} \frac{d \ell_{1} d \ell_{2}}{\left\|x_{\mathcal{C}}^{(1)}-x_{\mathcal{C}}^{(2)}\right\|}
$$

even though, in the case that $\mathbb{E}_{1}$ has identically vanishing eccentricity and $\mathcal{C}$ is regular in this limit, $h_{12}$ and $\bar{h}_{12}$ coincide. 
We shall prove the following formula. We let $\mathrm{U}$ as in (46) and

$$
\begin{aligned}
& \mathbb{E}\left(\mathrm{r}_{1}\right)=\sqrt{\mathrm{e}_{2, \mathcal{C}}^{2}+\mathrm{r}_{1} \mathrm{e}_{2, \mathcal{C}} \frac{\mathrm{C}_{\mathcal{C}}^{(1)} \cdot \mathrm{P}_{\mathcal{C}}^{(2)}}{\left\|\mathrm{C}_{\mathcal{C}}^{(1)}\right\| a_{2}}} \\
& \mathbb{I}\left(\mathrm{r}_{1}\right)=\sqrt{\frac{\left\|\mathrm{C}_{\mathcal{C}}^{(1)}\right\|^{2}\left\|\mathrm{C}_{\mathcal{C}}^{(2)}\right\|^{2}-\left(\mathrm{C}_{\mathcal{C}}^{(1)} \cdot \mathrm{C}_{\mathcal{C}}^{(2)}\right)^{2}}{\Lambda_{2}^{2}\left\|\mathrm{C}_{\mathcal{C}}^{(1)}\right\|^{2}}-\mathrm{r}_{1} \mathrm{e}_{2, \mathcal{C}} \frac{\mathrm{C}_{\mathcal{C}}^{(1)} \cdot \mathrm{P}_{\mathcal{C}}^{(2)}}{\left\|\mathrm{C}_{\mathcal{C}}^{(1)}\right\| a_{2}}}
\end{aligned}
$$

where the sub-fix $\mathcal{C}$ denotes the composition with $\mathcal{C}$. Then

Proposition $11 h_{12}=\left.\Pi_{\mathrm{r}_{1}} \mathrm{U}\left(\mathrm{r}_{1}, \mathrm{a}_{2}, \mathbb{E}\left(\mathrm{r}_{1}\right), \mathbb{I}\left(\mathrm{r}_{1}\right)\right)\right|_{\mathrm{r}_{1}=\left\|x_{\mathcal{C}}^{(1)}\right\|}$.

Remark 7 (Herman resonance for $h_{12}$ ) The functions $\mathbb{E}\left(\mathrm{r}_{1}\right), \mathbb{I}\left(\mathrm{r}_{1}\right)$ in (66) vanish, respectively, in case of zero eccentricity of the exterior planet and mutual inclination. Combining Propositions 7, 11, Remark 4, we obtain an eccentricity-inclination expansion for $h_{12}$ :

$$
h_{12}=\left.\sum_{h, k} \Pi_{\mathrm{r}_{1}}\left(\frac{\mathrm{r}_{1}^{2} a_{2}^{2} p_{h k}\left(\mathrm{r}_{1}, a_{2}\right)}{q\left(\mathrm{r}_{1}, a_{2}\right)^{\frac{1}{2}+2(h+k)}} \mathbb{E}\left(\mathrm{r}_{1}\right)^{2 h} \mathbb{I}\left(\mathrm{r}_{1}\right)^{2 k}\right)\right|_{\mathrm{r}_{1}=\left\|x_{\mathcal{C}}^{(1)}\right\|} .
$$

The second-order term of this expansion of course exhibits (43), as a by-product of Proposition 7 (because $\Pi_{\mathrm{r}}$ kills the linear terms in $\mathrm{r}_{1}$ in (66) acts on the even terms only modifying the coefficients). This identity reduces to the classical Herman resonance switching to Poincaré coordinates with the inner body moving on a circle. In this framework, Herman resonance naturally appears as a by-product of parities (39), renormalizable integrability of the Newtonian potential (Proposition 8), Keplerian property (Proposition 1) and Lemma 2.

To prove Proposition 11, we need an equivalent formulation of Lemma 2, which is as follows.

Proposition 12 Let $\mathrm{r}_{1}>0, \varphi_{1} \in \mathbb{T}, \mathrm{N}^{(1)} \in \mathbb{R}^{3}$, with $\left\|\mathrm{N}^{(1)}\right\|=1, z^{(2)} \in \mathbb{R}^{3}$, with $z^{(2)} \neq 0$, $z^{(2)} \nVdash \mathrm{N}^{(1)}$. Define $v:=z^{(2)} \times \mathrm{N}^{(1)}$. Let $z^{(1)}\left(\mathrm{r}_{1}, \varphi_{1}, \mathrm{~N}^{(1)}, z^{(2)}\right)$ be such that $z^{(1)} \perp \mathrm{N}^{(1)}$, $\left\|z^{(1)}\right\|=\mathrm{r}_{1}$ and $\alpha_{\mathrm{N}^{(1)}}\left(\nu, \mathrm{N}^{(1)} \times z^{(1)}\right)=\varphi_{1}$. Then, the following identity holds

$$
\frac{1}{2 \pi} \int_{\mathbb{T}} \frac{d \varphi_{1}}{\left\|z^{(1)}\left(\mathrm{r}_{1}, \varphi_{1}, \mathrm{~N}^{(1)}, z^{(2)}\right)-z^{(2)}\right\|}=\left.\frac{1}{\mathrm{r}_{2}} \Pi_{\varepsilon} \frac{1}{\left\|\varepsilon \mathrm{N}^{(1)}-\widetilde{z}^{(2)}\right\|}\right|_{\varepsilon=\frac{\mathrm{r}_{1}}{\mathrm{r}_{2}}},
$$

with $\mathrm{r}_{2}:=\left\|z^{(2)}\right\|, \widetilde{z}^{(2)}:=\frac{z^{(2)}}{\mathrm{r}_{2}}$. Such identity still holds replacing $z^{(1)}\left(\mathrm{r}_{1}, \varphi_{1}, \mathrm{~N}^{(1)}, z^{(2)}\right)$ with $z^{(1)}\left(\mathrm{r}_{1}, \varphi_{1}+\hat{\varphi}, \mathrm{N}^{(1)}, z^{(2)}\right)$, with any $\hat{\varphi}$, independent of $\varphi_{1}$.

Proof Let us decompose

$$
z^{(2)}=\left(z^{(2)} \cdot \mathrm{N}^{(1)}\right) \mathrm{N}^{(1)}+z_{\perp}^{(2)}
$$

where $z_{\perp}^{(2)}:=z^{(2)}-\left(z^{(2)} \cdot \mathrm{N}^{(1)}\right) \mathrm{N}^{(1)}$ is orthogonal to $\mathrm{N}^{(1)}$. Since $z^{(1)}$ is orthogonal to $\mathrm{N}^{(1)}$ and $\left\|z_{\perp}^{(2)}\right\|=\sqrt{\left\|z^{(2)}\right\|^{2}-\left(z^{(2)} \cdot \mathrm{N}^{(1)}\right)^{2}} \|=\mathrm{r}_{2} \sqrt{1-\left(\widehat{z}^{(2)} \cdot \mathrm{N}^{(1)}\right)^{2}}$, we have

$$
z^{(1)} \cdot z^{(2)}=z^{(1)} \cdot z_{\perp}^{(2)}=\left\|z^{(1)}\right\|\left\|z_{\perp}^{(2)}\right\| \cos \psi=\mathrm{r}_{1} \mathrm{r}_{2} \sqrt{1-\left(\widehat{z}^{(2)} \cdot \mathrm{N}^{(1)}\right)^{2}} \cos \psi
$$

where $\psi$ is the convex angle formed by $z^{(1)}$ and $z_{\perp}^{(2)}$. But $\psi$ is related to $\varphi_{1}$ via

$$
\psi=\left\|\pi-\varphi_{1}\right\|
$$


and therefore, $\cos \psi=-\cos \varphi_{1}$. This readily implies

$$
\left\|z^{(1)}\left(\mathrm{r}_{1}, \varphi_{1}, \mathrm{~N}^{(1)}, z^{(2)}\right)-z^{(2)}\right\|=\sqrt{\mathrm{r}_{1}^{2}+2 \mathrm{r}_{1} \mathrm{r}_{2} \sqrt{1-\left(\mathrm{N}^{(1)} \cdot \widehat{z}^{(2)}\right)^{2}} \cos \varphi_{1}+\mathrm{r}_{2}^{2}} .
$$

We now use this in the expansion of the inverse distance

$$
\frac{1}{\mathrm{D}\left(\mathrm{r}_{1}, \varphi_{1}, \mathrm{~N}^{(1)}, z^{(2)}\right)}=\frac{1}{\sqrt{\mathrm{r}_{1}^{2}+2 \mathrm{r}_{1} \mathrm{r}_{2} \sqrt{1-\left(\mathrm{N}^{(1)} \cdot \widehat{z}^{(2)}\right)^{2}} \cos \varphi_{1}+\mathrm{r}_{2}^{2}}}
$$

in terms of Legendre polynomials

$$
\frac{1}{\mathrm{D}\left(\mathrm{r}_{1}, \varphi_{1}, \mathrm{~N}^{(1)}, z^{(2)}\right)}=\frac{1}{\mathrm{r}_{2}} \sum_{n=0}^{\infty}(-1)^{n}\left(\frac{\mathrm{r}_{1}}{\mathrm{r}_{2}}\right)^{n} \mathcal{P}_{n}\left(\sqrt{1-\frac{\left(z^{(2)} \cdot \mathrm{N}^{(1)}\right)^{2}}{\mathrm{r}_{2}^{2}}} \cos \varphi_{1}\right) .
$$

To conclude, we only need to use Lemma 2, so that

$$
\frac{1}{2 \pi} \int_{\mathbb{T}} \mathcal{P}_{n}\left(\sqrt{1-\frac{\left(z^{(2)} \cdot \mathrm{N}^{(1)}\right)^{2}}{\mathrm{r}_{2}^{2}}} \cos \varphi_{1}\right) d \varphi_{1}=\delta_{n} P_{n}\left(\frac{z^{(2)} \cdot \mathrm{N}^{(1)}}{\mathrm{r}_{2}}\right),
$$

which is a rewrite of the thesis. From the formulae from (68) on, it follows that identity (67) still holds replacing $z^{(1)}\left(\mathrm{r}_{1}, \varphi_{1}, \mathrm{~N}^{(1)}, z^{(2)}\right)$ with $z^{(1)}\left(\mathrm{r}_{1}, \varphi_{1}+\hat{\varphi}, \mathrm{N}^{(1)}, z^{(2)}\right)$, for any $\hat{\varphi}$ independent of $\varphi_{1}$.

We can now proceed to prove Proposition 11. We do it in three steps.

First Step. Application of Proposition 12. Let $\mathcal{C}$ and $v_{1} \in \mathbb{R}^{3} \backslash\{0\}$ be as said at the beginning of this section. As a first step, we aim to compute the $g_{1}$-average applying Proposition 12 . If

$$
\mathrm{N}^{(1)}:=\frac{\mathrm{C}_{\mathcal{C}}^{(1)}}{\left\|\mathrm{C}_{\mathcal{C}}^{(1)}\right\|}, \quad v:=x_{\mathcal{C}}^{(2)} \times \mathrm{N}_{\mathcal{C}}^{(1)}
$$

then

$$
\alpha_{\mathrm{N}^{(1)}}\left(v, \mathrm{~N}^{(1)} \times x_{\mathcal{C}}^{(1)}\right)=\mathrm{g}_{1}+v_{1}+\frac{\pi}{2}-\hat{v} \text { where } \hat{v}=\alpha_{\mathrm{C}^{(1)}}\left(v_{1}, v\right)
$$

Hence, we can write

$$
x_{\mathcal{C}}^{(1)}=z^{(1)}\left(\left\|x_{\mathcal{C}}^{(1)}\right\|, \frac{\mathrm{C}_{\mathcal{C}}^{(1)}}{\left\|\mathrm{C}_{\mathcal{C}}^{(1)}\right\|}, \mathrm{g}_{1}+v_{1}+\frac{\pi}{2}-\hat{v}, x_{\mathcal{C}}^{(2)}\right),
$$

where $z^{(1)}$ is as in Proposition 12. We apply Proposition 12 with this $z^{(1)}, z^{(2)}=x_{\mathcal{C}}^{(2)}$, $\hat{\varphi}=v_{1}+\frac{\pi}{2}-\hat{v}$, which is independent of $\mathrm{g}_{1}$, by assumption. We find

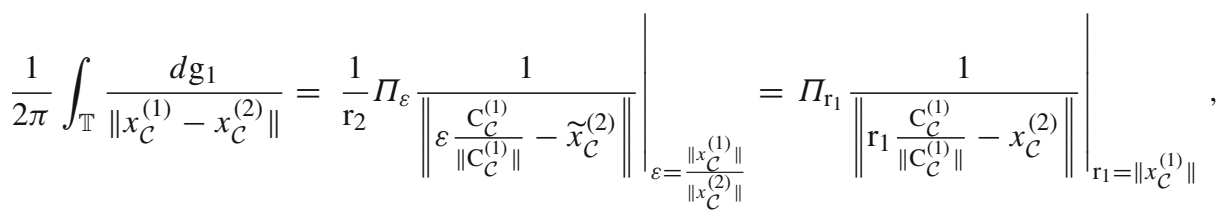


with $\tilde{x}_{\mathcal{C}}^{(2)}:=\frac{x_{\mathcal{C}}^{(2)}}{\left\|x_{\mathcal{C}}^{(2)}\right\|}$. Now we average with respect to $\ell_{2}$. We obtain, interchanging $\Pi_{\mathrm{r}_{1}}$ and $\int_{\mathbb{T}} d \ell_{2}$

$$
\frac{1}{4 \pi^{2}} \int_{\mathbb{T}^{2}} \frac{d \mathrm{~g}_{1} d \ell_{2}}{\left\|x_{\mathcal{C}}^{(1)}-x_{\mathcal{C}}^{(2)}\right\|}=\left.\frac{1}{2 \pi} \Pi_{\mathrm{r}_{1}} \int_{\mathbb{T}} \frac{d \ell_{2}}{\left\|\mathrm{r}_{1} \frac{\mathrm{C}_{\mathcal{C}}^{(1)}}{\left\|\mathrm{C}_{\mathcal{C}}^{(1)}\right\|}-x_{\mathcal{C}}^{(2)}\right\|}\right|_{\mathrm{r}_{1}=\left\|x_{\mathcal{C}}^{(1)}\right\|}
$$

Second Step. Switch to Delaunay coordinates. We apply $\phi_{\mathcal{C}}^{\mathcal{D}}$ in (65) to (69). We obtain

$$
\begin{aligned}
\left(\frac{1}{4 \pi^{2}} \int_{\mathbb{T}^{2}} \frac{d \mathrm{~g}_{1} d \ell_{2}}{\left\|x_{\mathcal{C}}^{(1)}-x_{\mathcal{C}}^{(2)}\right\|}\right) \circ \phi_{\mathcal{D}}^{\mathcal{C}} & =\frac{1}{2 \pi}\left(\left.\Pi_{\mathrm{r}_{1}} \int_{\mathbb{T}} \frac{d \ell_{2}}{\left\|\mathrm{r}_{1, \mathcal{C}} \frac{\mathrm{C}_{\mathcal{C}}^{(1)}}{\left\|\mathrm{C}_{\mathcal{C}}^{(1)}\right\|}-x_{\mathcal{C}}^{(2)}\right\|}\right|_{\mathrm{r}_{1}=\left\|x_{\mathcal{C}}^{(1)}\right\|} \circ \phi_{\mathcal{D}}^{\mathcal{C}}\right. \\
& =\left.\frac{1}{2 \pi} \Pi_{\mathrm{r}_{1}} \int_{\mathbb{T}} \frac{d \mathrm{l}_{2}}{\left\|\mathrm{r}_{1, \mathcal{D}} \frac{\mathrm{C}_{\mathcal{D}}^{(1)}}{\left\|\mathrm{C}_{\mathcal{D}}^{(1)}\right\|}-x_{\mathcal{D}}^{(2)}\right\|}\right|_{\mathrm{r}_{1}=\left\|x_{\mathcal{D}}^{(1)}\right\|} \\
& =\mathrm{U}\left(\mathrm{r}_{1, \mathcal{D}}, a_{2}, \mathcal{E}_{\left.2, \mathcal{D}_{2}, \mathcal{I}_{2, \mathcal{D}_{2}}\right),}\right.
\end{aligned}
$$

where $\mathcal{E}_{2, \mathcal{D}_{2}}, \mathcal{I}_{2, \mathcal{D}_{2}}$ are as in $(50)$, with $\mathcal{C}_{2}=\mathcal{D}_{2}$. We have that $\mathrm{r}_{1, \mathcal{D}} \frac{\mathrm{C}_{\mathcal{D}}^{(1)}}{\left\|\mathrm{C}_{\mathcal{D}}^{(1)}\right\|}$ depends only on $\mathcal{D}_{1}=\left(\Lambda_{1}, 1_{1}, p_{1}, q_{1}\right)$, while $x_{\mathcal{D}}^{(2)}$ depends only on $\mathcal{D}_{2}=\left(\Lambda_{2}, 1_{2}, p_{2}, q_{2}\right)$. We have used Proposition 9 with a given $\widetilde{w} \in \mathbb{R}^{3}, \mathcal{C}_{2}=\mathcal{D}_{2}$ and next we have taken $\widetilde{w}=\mathrm{r}_{1, \mathcal{D}} \frac{\mathrm{C}_{\mathcal{D}}^{(1)}}{\left\|\mathrm{C}_{\mathcal{D}}^{(1)}\right\|}$.

Third Step. Applying $\left(\phi_{\mathcal{C}}^{\mathcal{D}}\right)^{-1}$, we conclude the proof.

\subsection{Is the two-centre Hamiltonian renormalizably integrable?}

In this section, we outline an underlying open problem in the framework of the paper. We pose a conjecture that we aim to study in further work, which, if proved, may be applied to the two-centre Hamiltonian (8), so as to obtain a stronger assertion than Proposition 6.

Throughout the section, $V \subset \mathbb{R}, \mathcal{U} \subset \mathbb{R}^{2}$ are domains, $(I, \varphi) \in \mathcal{I} \times \mathbb{T},(p, q) \in \mathcal{U}$ are pairwise conjugate canonical coordinates. We shall be concerned with real-analytic ${ }^{9}$ functions ("Hamiltonians") for $(I, \varphi, p, q, \mu) \in \mathcal{P}=\mathcal{I} \times \mathbb{T} \times \mathcal{U} \times\left(-\mu_{0}, \mu_{0}\right)$ having the form:

$$
h=h_{0}(I)+\mu f(I, \varphi, p, q, \mu) \quad \text { with } \quad h_{0}(I) \not \equiv 0 \text { on } V .
$$

Definition 4 We say that $\bar{h}$ is in p-normal form if there exist $\left\{\bar{h}_{k}(\bar{I}, \bar{p}, \bar{q})\right\}_{k=0, \ldots, p} h_{k}$ : $V \times \mathcal{U} \rightarrow \mathbb{R}$ such that

$$
\bar{h}(\bar{I}, \bar{\varphi}, \bar{p}, \bar{q})=\sum_{k=0}^{p} \bar{h}_{k}(\bar{I}, \bar{p}, \bar{q}) \mu^{k}+\mathrm{O}\left(\mu^{p+1}\right) \quad \forall(\bar{I}, \bar{\varphi}, \bar{p}, \bar{q}) \in \mathcal{P} .
$$

The following result is well known and hence will be not discussed.

\footnotetext{
${ }^{9}$ Following the standard terminology, a real function $h$ is said to be real-analytic on a domain $\mathcal{P} \subset \mathbb{R}^{p}$ if there exists an open set $\hat{\mathcal{P}}$, with $\mathcal{P} \subset \hat{\mathcal{P}} \subset \mathbb{C}^{p}$, such that $h$ has a holomorphic extension on $\hat{\mathcal{P}}$.
} 
Proposition 13 Let $h$ be as in (70). For any $p \in \mathbb{N}$, it is possible to find a real-analytic, canonical and $\mu$-close to the identity transformation

$$
\phi:(\bar{I}, \bar{\varphi}, \bar{p}, \bar{q}) \in \mathcal{P} \rightarrow(I, \varphi, p, q) \in \mathcal{P}
$$

such that $\bar{h}:=h \circ \phi$ is in $p$-normal form:

$$
\bar{h}(\bar{I}, \bar{\varphi}, \bar{p}, \bar{q})=\sum_{k=0}^{p} \bar{h}_{k}(\bar{I}, \bar{p}, \bar{q}) \mu^{k}+\mathrm{O}\left(\mu^{p+1}\right) \quad \forall(\bar{I}, \bar{\varphi}, \bar{p}, \bar{q}) \in \mathcal{P},
$$

with $\bar{h}_{0}(\bar{I}, \bar{p}, \bar{q})=h_{0}(\bar{I})$.

Lemma 4 Let $h$ be in p-normal form and let $g$ be a first integral of $h$. Then

(i) $g$ is in p-normal form;

(ii) $\left\{\bar{h}_{1}, \bar{g}\right\}=\mathrm{O}\left(\mu^{p}\right)$, where $\bar{h}_{1}(I, p, q, \mu):=\sum_{k=1}^{p} \bar{h}_{k}(\bar{I}, \bar{p}, \bar{q}) \mu^{k}$.

Proof (i) Let

$$
g(I, \varphi, p, q, \mu)=\sum_{k=0}^{\infty} \bar{g}_{k}(I, \varphi, p, q) \mu^{k}
$$

denote the Taylor-Maclaurin series in $\mu$ of $g$. We prove that the functions $\bar{g}_{j}$ are $\varphi$ independent for all $0 \leq j \leq p$. We proceed by induction on $j$. Since $h(\cdot, \cdot, \cdot, \cdot, \mu)$ and $g(\cdot, \cdot, \cdot, \cdot, \mu)$ Poisson commute for all $\mu \in\left(-\mu_{0}, \mu_{0}\right)$, we find

$$
\left\{h_{0}, \bar{g}_{0}\right\}=\partial_{I} h_{0}(I) \partial_{\varphi} \bar{g}_{0}(I, \varphi, p, q) \equiv 0,
$$

where we have used that $h_{0}$ depends only on $I$. Since, by assumption, $\partial_{I} h_{0}(I) \not \equiv 0$, it follows that $\partial_{\varphi} \bar{g}_{0}(I, \varphi, p, q) \equiv 0$ and hence $\bar{g}_{0}(I, \varphi, p, q)$ is $\varphi$-independent, and hence $\bar{g}_{0}(I, \varphi, p, q)=\bar{g}_{0}(I, 0, p, q)=g_{0}(I,(p, q))$ for all $\varphi \in \mathbb{T}$, with $g_{0}(I,(p, q))$ as in (ii). So the step $j=0$ is proved. Assume now that, for a given $0 \leq j<p$ and any $0 \leq k \leq j, \bar{g}_{k}$ is $\varphi$-independent. Namely, $\bar{g}_{k}(I, \varphi, p, q)=g_{k}(I,(p, q))$, for some function $g_{k}(I,(p, q))$, with $0 \leq k \leq j$. We prove that $\bar{g}_{j+1}$ is so. Since $h$ and $g$ Poisson commute,

$$
\{\bar{h}, \bar{g}\}=\mathrm{O}\left(\mu^{p+1}\right) .
$$

Since $j+1 \leq p$, the projection of the left-hand side over the monomial $\mu^{j+1}$ vanishes:

$$
\left\{h_{0}, \bar{g}_{j+1}\right\}+\sum_{k=0}^{j}\left\{h_{j-k+1}, g_{k}\right\}=0 .
$$

In this identity, the term $\left\{h_{0}, \bar{g}_{j+1}\right\}$ has vanishing $\varphi$-average, because $h_{0}$ depends only on $I$, while the term $\sum_{k=0}^{j}\left\{h_{j-k+1}, g_{k}\right\}$ is $\varphi$-independent, due to the fact that the $h_{j-k+1}$ (by assumption) and the $g_{k}$ (by the inductive hypothesis) are so. Therefore, such two terms have to identically vanish separately:

$$
\left\{h_{0}, \bar{g}_{j+1}\right\} \equiv 0 \equiv \sum_{k=0}^{j}\left\{h_{j-k+1}, g_{k}\right\}
$$


The vanishing of the left-hand side implies, as in the base step, that $\bar{g}_{j+1}$ is $\varphi$-independent. The vanishing of the right-hand side for all $0 \leq j+1 \leq p$ is a rewrite of ${ }^{10}$ (ii).

Corollary 1 For any $p$,

$$
\bar{g}_{\text {tr }}^{(p)}(I, p, q)=g_{0}(I, p, q)+\sum_{k=1}^{p-1} \bar{g}_{k}(I, p, q) \mu^{k}, \quad \bar{h}_{1, \mathrm{tr}}^{(p)}(I, p, q)=\sum_{k=0}^{p-1} \bar{h}_{k+1}(I, p, q) \mu^{k}
$$

verify

$$
\left\{\bar{g}_{\mathrm{tr}}^{(p)}, \bar{h}_{1, \mathrm{tr}}^{(p)}(I, p, q)\right\}=\mathrm{O}\left(\mu^{p}\right) .
$$

We recall that

$$
\bar{h}_{1}(\bar{I}, \bar{p}, \bar{q}, 0)=\frac{1}{2 \pi} \int_{0}^{2 \pi} h_{1}(\bar{I}, \varphi, \bar{p}, \bar{q}, 0) d \varphi .
$$

Definition 5 We shall refer to the formal series $\sum_{k=0}^{\infty} \bar{h}_{k}(\bar{I}, \bar{p}, \bar{q}) \mu^{k}$ as perturbative series in $\mu$ to $h$.

Conjecture 1 If $h$ as in (70) has an independent first integral, its perturbative series converges, as well as the perturbative series to $g$. If $\bar{h}, \bar{g}$ denote the sum of the two series, $\bar{h}$ is renormalizably integrable via $\bar{g}$.

\section{Compliance with ethical standards}

Conflicts of interest The author declares that she has no conflict of interest.

Open Access This article is distributed under the terms of the Creative Commons Attribution 4.0 International License (http://creativecommons.org/licenses/by/4.0/), which permits unrestricted use, distribution, and reproduction in any medium, provided you give appropriate credit to the original author(s) and the source, provide a link to the Creative Commons license, and indicate if changes were made.

\section{References}

Abdullah, K., Albouy, A.: On a strange resonance noticed by M. Herman. Regul. Chaotic Dyn. 6(4), 421-432 (2001)

Arnold, V.I.: Small denominators and problems of stability of motion in classical and celestial mechanics. Russ. Math. Surv. 18(6), 85-191 (1963)

Bekov, A.A., Omarov, T.B.: Integrable cases of the Hamilton-Jacobi equation and some nonsteady problems of celestial mechanics. Sov. Astron. 22, 366-370 (1978)

Chierchia, L., Pinzari, G.: The planetary $N$-body problem: symplectic foliation, reductions and invariant tori. Invent. Math. 186(1), 1-77 (2011)

Deprit, A.: Elimination of the nodes in problems of $n$ bodies. Celest. Mech. 30(2), 181-195 (1983)

Féjoz, J.: Démonstration du 'théorème d'Arnold' sur la stabilité du système planétaire (d'après Herman). Ergod. Theory Dyn. Syst. 24(5), 1521-1582 (2004)

10 Alternatively, observe that, since $\bar{g}$ is independent of $\varphi$ up to the order $\mathrm{O}\left(\mu^{p+1}\right)$ and $h, g$ do Poisson commute, by (71),

$$
\mu\left\{\bar{h}_{1}, \bar{g}\right\}=\left\{h_{0}, \bar{g}\right\}+\mu\left\{\bar{h}_{1}, \bar{g}\right\}+\mathrm{O}\left(\mu^{p+1}\right)=\{\bar{h}, \bar{g}\}+\mathrm{O}\left(\mu^{p+1}\right)=\{h, g\}+\mathrm{O}\left(\mu^{p+1}\right)=\mathrm{O}\left(\mu^{p+1}\right) .
$$


Féjoz, J.: Quasi periodic motion in the planar three-body problem. J. Differ. Equ. 183, 303-341 (2002)

Féjoz, J.: On "Arnold's theorem" in celestial mechanics -a summary with an appendix on the Poincaré coordinates. Discrete Contin. Dyn. Syst. 3, 3555-3565 (2013)

Féjoz, J., Guardia, M.: Secular instability in the three-body problem. Arch. Ration. Mech. Anal. 221(1), 335-362 (2016)

Gallavotti, G.: Phénomènes critiques, systèmes aléatoires, théories de jauge, Quasi-integrable mechanical systems, pp. 539-624, Amsterdam (1986)

Giorgilli, A.: Appunti di Meccanica Celeste. http://www.mat.unimi.it/users/antonio/meccel/meccel.html (2008)

Harrington, R.S.: The stellar three-body problem. Celestial Mech. 1(2), 200-209 (1969)

Jacobi, C.G.J.: Sur l'élimination des nœuds dans le problème des trois corps. Astron. Nachr. Bd XX, 81-102 (1842)

Laskar, J., Robutel, P.: Stability of the planetary three-body problem. I. Expansion of the planetary Hamiltonian. Celest. Mech. Dyn. Astron. 62(3), 193-217 (1995)

Meyer, K.R., Palacián, J.F., Yanguas, P.: Singular reduction of resonant Hamiltonians. Nonlinearity 31(6), 2854-2894 (2018)

Palacián, J.F., Sayas, F., Yanguas, P.: Regular and singular reductions in the spatial three-body problem. Qual. Theory Dyn. Syst. 12(1), 143-182 (2013)

Palacián, J.F., Vanegas, J., Yanguas, P.: Compact normalisations in the elliptic restricted three body problem. Astrophys. Space Sci. 362, 215 (2017)

Pinzari, G.: Canonical coordinates for the planetary problem. Acta Appl. Math. 137(1), 205-232 (2015)

Pinzari, G.: Exponential stability of Euler integral in the three-body problem. arXiv:1808.07633 (2018)

Pinzari, G.: Perihelia reduction and global Kolmogorov tori in the planetary problem. Mem. Am. Math. Soc. 255, 1218 (2018)

Sundman, K.F.: Sur les conditions nécessaires et suffisantes pour la convergence du développement de la fonction perturbatrice dans le mouvement plan. Öfvers af FVS. förh 58, A:24 (1916)

Publisher's Note Springer Nature remains neutral with regard to jurisdictional claims in published maps and institutional affiliations. 